\title{
The Quantum Group Structure of 2D Gravity and Minimal Models I.
}

\author{
Jean-Loup Gervais \\ Laboratoire de Physique Théorique de l'École Normale Supérieure, ${ }^{\star} 24$, rue Lhomond, F-75231 Paris \\ Cedex 05, France
}

\begin{abstract}
On the unit circle, an infinite family of chiral operators is constructed, whose exchange algebra is given by the universal $R$-matrix of the quantum group $S L(2)_{q}$. This establishes the precise connection between the chiral algebra of two dimensional gravity or minimal models and this quantum group. The method is to relate the monodromy properties of the operator differential equations satisfied by the generalized vertex operators with the exchange algebra of $S L(2)_{q}$. The formulae so derived, which generalize an earlier particular case worked out by Babelon, are remarkably compact and may be entirely written in terms of " $q$-deformed" factorials and binomial coefficients.
\end{abstract}

\section{Introduction}

Quantum groups came out from the study of conformally invariant field theories and integrable models [1-3]. In particular, Neveu and I [2,4] already wrote an exchange algebra associated with $S L(2)_{q}$ as early as 1983 , by studying the monodromy properties of the differential equation for chiral vertex operators $[5,6]$. Our formulae did not look like the standard ones since they came out in a different basis, but recently Babelon [3] explicitly established the connection for the simplest set of operators in the chiral conformal family. Our results of ref. [2] concerning the monodromy of conformal blocks have been rediscovered and generalized later on $[7,8]$. However, apart from ref. [3], their precise connection with the quantum group $S L(2)_{q}$ remains to be established. Such is the aim of the present paper.

In an earlier work [4], we had devised a formalism, in order to handle the chiral conformal Virasoro algebra, that is formulated in terms of two free fields which play a symmetric role, contrary to the more standard Coulomb gas picture. As we shall see this point is crucial. It is most simply explained by looking first at the following classical structure which came out of our studies $[2,4,5,9]$ and

* Laboratoire Propre du Centre National de la Recherche Scientifique, associé à l'École Normale Supérieure et à l'Université de Paris-Sud 
is of central importance in 2D gravity and minimal models. Consider a classical $c$-number field $p(\sigma)$, defined on the unit circle $(0 \leqq \sigma \leqq 2 \pi)$, which satisfies the Poisson bracket (P.B.) relations

$$
\left\{p\left(\sigma_{1}\right), p\left(\sigma_{2}\right)\right\}=2 \pi \delta^{\prime}\left(\sigma_{1}-\sigma_{2}\right) .
$$

Primes denote $\sigma$-derivatives. Let $\gamma$ be a free parameter that plays the role of the coupling constant, and introduce the field

$$
T(\sigma) \equiv \gamma p^{2}(\sigma)+\sqrt{\gamma} p^{\prime}(\sigma) .
$$

It easily follows from (1.1) that if we define

$$
L_{n} \equiv \frac{1}{4 \pi \gamma} \int_{0}^{2 \pi} d \sigma T(\sigma) e^{i n \sigma}+\frac{1}{8 \gamma} \delta_{n, 0}
$$

we have a P.B. realization of the Virasoro algebra:

$$
i\left\{L_{m}, L_{n}\right\}=(m-n) L_{m+n}+\frac{C}{12}\left(m^{3}-m\right) \delta_{m,-n},
$$

where $C=3 / \gamma$. The above expression may be rewritten as:

$$
T / \gamma=\left(\phi^{\prime}\right)^{2}+\phi^{\prime \prime} / \sqrt{\gamma} \quad \phi^{\prime} \equiv p,
$$

which is a chiral component of a deformed $U(1)$ Sugawara stress-energy tensor. This simple realization of the Virasoro algebra came out in a very natural way from the canonical treatment of the Liouville theory [2,4,5,9]. It is the basis of the Coulomb gas picture of conformal theories. In this connection Neveu and I have pointed out [4] that the relationship between $p$ and $T$ so defined, which coincides with the so-called Miura transformation of the KdV system, is not one-to-one but two-to-one. Consider the equality (1.2), with a given function $T(\sigma)$ as an equation for the field $p(\sigma)$. From this viewpoint it is an equation of the Riccati type which is well-known to have two independent solutions. This becomes obvious if we let $p \equiv \psi^{\prime} /(\psi \sqrt{\gamma})$, obtaining the Schrödinger equation

$$
-\psi^{\prime \prime}+T \psi=0
$$

Denote by $\psi_{j}, j=1,2$, two independent solutions of (1.6). Going backward one obtains two $p$-fields, $p_{j} \equiv \psi_{j}^{\prime} /\left(\psi_{j} \sqrt{\gamma}\right), j=1,2$, which are such that

$$
T / \gamma=p_{1}^{2}+p_{1}^{\prime} / \sqrt{\gamma}=p_{2}^{2}+p_{2}^{\prime} / \sqrt{\gamma} .
$$

Thus the correspondence between $p$ and $T$ is two-to-one as already stated. Since the Schrödinger equation is linear, the $\psi$-fields are determined up to linear combinations. The potential $T$ of Eq. (1.6) being periodic in $\sigma$, one has $\psi_{i}(\sigma+2 \pi)=\sum_{j} M_{i}^{j} \psi_{j}(\sigma)$. Generically one may diagonalize the monodromy matrix $M_{i}^{j}$ so that the Schrödinger wave functions are periodic up to multiplicative constants (Bloch waves). With this choice the $p_{j}$ 's are periodic and one may write:

$$
p_{j}=\sum_{n} e^{-i n \sigma} p_{n}^{(j)} \text {. }
$$


For later use we note that it is convenient $[2,5]$ to normalize the Schrödinger wave functions as

$$
\psi_{j} \equiv d_{j} e^{\sqrt{\gamma} \phi_{j}} \quad \text { with } \quad \phi_{j}(\sigma)=q_{0}^{(j)}+p_{0}^{(j)} \sigma+i \sum_{n \neq 0} \frac{e^{-i n \sigma}}{n} p_{n}^{(j)},
$$

where the $d_{j}$ are integration constant that only depends upon $p_{0}^{(j)}$. The variable $q_{0}^{(j)}$ is assumed to satisfy the P.B. relations ${ }^{\dagger}$

$$
\left\{q_{0}^{(j)}, p_{0}^{(j)}\right\}=1
$$

so that the $\psi$ 's are classical vertex functions similar to the standard string vertex, $q_{0}^{(j)}$ and $p_{0}^{(j)}$ playing the role of center of mass positions and total momenta respectively. Equation (1.10) ensures, in particular, that the $\psi$ 's satisfy the P.B. relations:

$$
i\left\{L_{n}, \psi_{j}(\sigma)\right\}=e^{i n \sigma}\left(-i \frac{d}{d \sigma}+n \Delta\right) \psi_{j}(\sigma) ; \quad \text { with } \quad \Delta=-\frac{1}{2} .
$$

They are thus classical primary fields of weight $-1 / 2$, that is, half differentials. (Since we work on the unit circle, I adopt the corresponding definition of primary fields: the coefficient of the $\Delta$-term is $n$ instead of the more standard $n+1$. The latter definition is recovered by mapping to the complex plane, since a primary field of weight $\Delta$ is multiplied by $e^{i \Delta \sigma}$ if one performs the conformal transformation $z=e^{i \sigma}$.)

It obviously follows from (1.9) that $\psi_{i}(\sigma+2 \pi)=e^{2 \pi p_{0}^{(i)}} \psi_{i}(\sigma)$. Standard Wronskian arguments show that the determinant of the monodromy matrix is one, so that the product of its eigenvalues is equal to one. Therefore

$$
p_{0}^{(1)}=-p_{0}^{(2)} \text {. }
$$

As is well known, $p_{0}^{(1)}=-p_{0}^{(2)}$ may be either real (in the so-called forbidden zones where the Schrödinger wave functions decay or blow up exponentially over a period), or pure imaginary (in the so-called allowed zones where the Schrödinger wave functions are oscillatory). There are special cases, at the border between allowed and forbidden zones where the monodromy matrix may not be diagonalizable. We shall not consider this possibility (more about this later on).

Concerning the P.B. structure, Neveu and I have shown that it is completely symmetric between $p_{1}$ and $p_{2}$, namely both satisfy (1.1):

$$
\left\{p_{1}\left(\sigma_{1}\right), p_{1}\left(\sigma_{2}\right)\right\}=\left\{p_{2}\left(\sigma_{1}\right), p_{2}\left(\sigma_{2}\right)\right\}=2 \pi \delta^{\prime}\left(\sigma_{1}-\sigma_{2}\right) .
$$

The P.B. relations between $p_{1}$ and $p_{2}$ are complicated and not very illuminating. The interesting structure is provided by the P.B. relations between the $\psi$ 's, which close in the sense that

$$
\left\{\psi_{i}\left(\sigma_{1}\right), \psi_{j}\left(\sigma_{2}\right)\right\}=\sum_{k=1,2 ; l=1,2} s_{i j}^{k l} \psi_{k}\left(\sigma_{1}\right) \psi_{l}\left(\sigma_{2}\right),
$$

\footnotetext{
${ }^{1}$ Throughout this article, no summation over repeated indices is to be performed unless explicitly indicated
} 
where $s_{i j}^{k l}$ only depends upon $p_{0}^{(1)}$ and plays the role of a classical $R$-matrix. In ref. [9] we remarked that letting $A \equiv \psi_{2} / \psi_{1}$ leads to $\psi_{1}=1 / \sqrt{A^{\prime}}, \psi_{2}=A / \sqrt{A^{\prime}}$, and that $-2 T$ is equal to the Schwarzian derivative of $A$. We worked out the P.B. algebra of the $A$-field (Eq. (3.34) of ref. [9]) taking account of the periodicity condition in $\sigma$. Equation (1.14) is an immediate consequence of this last P.B. structure. Its precise form will be given later on since it depends upon the choice of the $d_{i}$ 's in Eq. (1.9). We shall see that it is related to a classical limit of the exchange algebra of $S L(2)_{q}$.

The field $T$ has conformal weight 2 up to a central term and defines a quadratic differential on the circle. The $p$-fields transform as a linear differentials up to central terms. Thus Eq. (1.2) may be regarded as defining linear differentials as square roots of the given quadratic differential $T$. The existence of two independent $p$-fields for a given $T$, that will play a crucial role in the following, is thus very natural. Exchanging $p_{1}$ and $p_{2}$ is a canonical transformation that plays the same role as the exchange of solutions of an algebraic equation. It is thus a generalized Galois transformation. As we shall see this transformation is the basis of the quantum group structure.

The results presented next follow the trend of my previous works with A. Neveu and A. Bilal which is quite different from the more common line initiated by BPZ [10]. Our main tools are operator differential equations which are derived without invoking the decoupling of null states. It is thus possible to work for general value of $\mathrm{C}$ and generic Verma modules that do not contain null vectors. In this situation, the transformation between $p_{1}$ and $p_{2}$ fully extends to the quantum case [4]. (This is the quantum equivalent of avoiding borders between allowed and forbidden zones which we mentioned above.) In this article, I only discuss this generic situation, which is also simpler because the corresponding representations of the quantum group have $q$-values not equal to a root of the unity, and are thus simple deformations of the standard group representations. In this philosophy, the rational theories are recovered by taking limits of the general formulae discussed below. I plan to retun to this in later publications.

\section{The Conformal Structure in the Block Wave Basis}

At the quantum level Neveu and I [4] have shown that the structure sketched above survives with little modifications and that, for generic $C$, the symmetry between $\phi_{1}$ and $\phi_{2}$ may be kept. One defines two normal ordering noted $N^{(1)}$ and $N^{(2)}$ that are with respect to the modes of $p_{1}$ and $p_{2}$ respectively. Equations (1.13, 17) are replaced by:

$$
\begin{aligned}
{\left[p_{1}\left(\sigma_{1}\right), p_{1}\left(\sigma_{2}\right)\right] } & =\left[p_{2}\left(\sigma_{1}\right), p_{2}\left(\sigma_{2}\right)\right]=2 \pi i \delta^{\prime}\left(\sigma_{1}-\sigma_{2}\right), \quad p_{0}^{(1)}=-p_{0}^{(2)}, \\
N^{(1)}\left(p_{1}^{2}+p_{1}^{\prime} / \sqrt{\gamma}\right) & =N^{(2)}\left(p_{2}^{2}+p_{2}^{\prime} / \sqrt{\gamma}\right) .
\end{aligned}
$$

The latter equation defines the parameter $\gamma$ at the quantum level. It is such that the central charge is given by $C=1+3 / \gamma$. Equation (1.9) becomes [2]

$$
\psi_{i}=d_{i} N^{(i)}\left(e^{\sqrt{h / 2 \pi} \phi_{i}}\right)
$$


where $^{2}$ there are two choices for the number $h$

$$
h_{ \pm}=\frac{\pi}{4 \gamma}(1-4 \gamma \pm \sqrt{1-8 \gamma})=\frac{\pi}{12}(C-13 \pm \sqrt{(C-25)(C-1)}) .
$$

For each $i=1,2$ there are thus two different fields $\psi_{i}^{ \pm}$. In the classification of BPZ [10] both $\psi_{1}^{+}$and $\psi_{2}^{+}$(respectively $\psi_{1}^{-}$and $\psi_{2}^{-}$) correspond to the $(1,2)$ (respectively $(2,1))$ chiral primary fields. In the standard Coulomb gas picture one only introduces one of the two free fields, say, $p_{1}$, and only $\psi_{1}^{ \pm}$is defined by (2.2). $\psi_{2}^{ \pm}$ are expressed in terms of $\psi_{1}^{ \pm}$and $p_{1}$ by means of the screening operators. From the quantum group viewpoint it is better to remain symmetric as we do here. For the time being we shall not consider the algebra that mixes the two choices of $h$. It is thus not necessary to distinguish the two values of $h$, and I drop the indices \pm . The complete algebra will be discussed in Sect. 4 .

Neveu and I $[2,5]$ long ago derived the exchange algebra of the $\psi$ fields from the monodromy properties of the quantum differential equation ${ }^{3}$ corresponding to (1.6). If one looks at the exchange of $\psi$ fields, our result is equivalent to:

with the condition:

$$
\psi_{i}(\sigma) \psi_{j}\left(\sigma^{\prime}\right)=\sum_{k=1,2 ; l=1,2} S_{i j}^{k l} \psi_{k}\left(\sigma^{\prime}\right) \psi_{l}(\sigma)
$$

$$
d_{1}(\omega) d_{2}(\omega-1)=\Gamma(-\omega h / \pi) \Gamma((\omega-1) h / \pi),
$$

that gives, as non-zero matrix elements,

$$
\begin{gathered}
S_{11}^{11}=S_{22}^{22}=e^{-i h \varepsilon / 2}, \\
S_{12}^{12}(\omega)=S_{21}^{21}(-\omega)=\frac{1}{\lfloor\omega\rfloor} e^{i h \varepsilon(1 / 2-\omega)}, \quad S_{12}^{21}(\omega)=S_{21}^{12}(-\omega)=\frac{\lfloor\omega+1\rfloor}{\lfloor\omega\rfloor} e^{i h \varepsilon / 2} .
\end{gathered}
$$

$\varepsilon$ is equal to the sign of $\sigma-\sigma^{\prime}, \omega$ is related to the zero mode of the free $p$-fields by the relation

$$
\omega=i p_{0}^{(1)} \sqrt{\frac{2 \pi}{h}},
$$

and we introduce the convenient notation:

$$
\lfloor x\rfloor \equiv \frac{\sin (h x)}{\sin (h)} .
$$

The above formulae are not quite the expression derived in ref. [2]. The latter involves $\Gamma$ functions. The $d_{i}$ 's are chosen such that they are replaced by trigonometric functions. Some more details are given in Appendix A.

Since they describe the exchange algebra of operators the above $S_{i j}^{k l}$ do satisfy the Yang-Baxter equations. However, they depend upon $\omega$ which is shifted by

\footnotetext{
${ }^{2}$ Warning! The notation is different from ref. [2]: $\gamma, h_{ \pm}$, and $h \omega$ here are equal to $\hbar, h \eta_{ \pm}^{2}$, and $\eta \omega$ there, respectively. Moreover, I have rescaled $p$ by $\sqrt{\gamma}$

${ }^{3}$ Which reflects the decoupling of Virasoro null vectors in the BPZ language
} 
the $\psi$ fields according to:

$$
\psi_{i} f(\omega)=f\left(\omega+\varepsilon_{i}\right) \psi_{i} ; \quad \varepsilon_{2}=-\varepsilon_{1}=1 .
$$

Thus the Yang-Baxter equations read [2]:

$$
\begin{gathered}
\sum_{\rho, \lambda, \mu} S_{i \lambda}^{l \rho}\left(\omega, \sigma_{1}-\sigma_{2}\right) S_{j k}^{\lambda \mu}\left(\omega+\varepsilon_{i}, \sigma_{2}-\sigma_{3}\right) S_{\rho \mu}^{m n}\left(\omega+\varepsilon_{l}, \sigma_{1}-\sigma_{2}\right) \\
=\sum_{\rho, \lambda, \mu} S_{i j}^{\mu \lambda}\left(\omega, \sigma_{1}-\sigma_{2}\right) S_{\lambda k}^{\rho n}\left(\omega+\varepsilon_{\mu}, \sigma_{1}-\sigma_{3}\right) S_{\mu \rho}^{l n}\left(\omega, \sigma_{2}-\sigma_{3}\right) .
\end{gathered}
$$

The basic point in deriving these properties is that the two-point functions of the $\psi$ fields satisfy hypergeometric differential equations. The exchange algebra is a consequence of the well-known relation (for $|\arg (-x)|<\pi$ ):

$$
\begin{aligned}
F(a, b ; c ; x)= & \frac{\Gamma(c) \Gamma(b-a)}{\Gamma(b) \Gamma(c-b)}(-x)^{-a} F\left(a, 1-c+a ; 1-b+a ; \frac{1}{x}\right) \\
& +\frac{\Gamma(c) \Gamma(a-b)}{\Gamma(a) \Gamma(c-b)}(-x)^{-b} F\left(b, 1-c+b ; 1-a+b ; \frac{1}{x}\right) .
\end{aligned}
$$

Similarly, one may determine the fusion rules of the $\psi$ fields to leading order from the relation (for $|\arg (1-x)|<\pi$ ):

$$
\begin{aligned}
F(a, b ; c ; x)= & \frac{\Gamma(c) \Gamma(c-b-a)}{\Gamma(c-a) \Gamma(c-b)} F(a, b ; a+b-c+1 ; 1-x) \\
& +\frac{\Gamma(c) \Gamma(a+b-c)}{\Gamma(a) \Gamma(b)}(1-x)^{c-a-b} F(c-a, c-b ; c-a-b+1 ; 1-x) .
\end{aligned}
$$

These results may be extended to more general operators, since the two-point function of one $\psi$ field with any primary field is also given by a hypergeometric function [2]. In this way one defines fields $\psi^{\mu, v}$ for arbitrary positive $\mu$ and $v$. (My collaborators and I have repeatedly stated the general features of this family $[5,11]$ without giving their explicit derivation. This is done, to some extent, in Appendix A.) In this connection it is simpler to temporarily restrict ourselves to the interval $1 / 8>\gamma>0$, that is $C>25$, where the leading term of the short distance operator product expansion (OPE) just corresponds to adding the $\mu$ 's and the $v^{\text {'s. In this }}$ way, for instance, one has $\psi^{\mu, v} \sim \psi_{1}^{\mu} \psi_{2}^{v}, \psi_{1}^{\mu} \propto N^{(1)}\left(e^{\mu \sqrt{h / 2 \pi} \phi_{1}}\right), \psi_{2}^{v} \propto N^{(2)}\left(e^{v \sqrt{h / 2 \pi} \phi_{2}}\right)$; where $\sim$ means that one takes the corresponding term in the short distance expansion and removes the standard divergent piece. Once the conformal family is derived one may continue to other values of $\gamma$. The term considered becomes non-leading but the construction remains valid.

The properties of the $\psi^{\mu, v}$ 's may be obtained recursively from the fusion rules:

$$
\psi_{1} \psi^{\mu, v} \sim \frac{\lfloor\omega+v\rfloor}{\lfloor\omega\rfloor} \psi^{\mu+1, v}, \quad \psi_{2} \psi^{\mu, v} \sim \frac{\lfloor\omega-\mu\rfloor}{\lfloor\omega\rfloor} \psi^{\mu, v+1}
$$

It is convenient to adopt a notation ispired by $S U(2)$. We let:

$$
\psi_{m}^{(J)} \equiv \psi^{J-m, J+m} ; \quad \mu+v=2 J, \quad v-\mu=2 m
$$


so that $2 m$ is equal to the shift:

$$
\psi_{m}^{(J)} f(\omega)=f(\omega+2 m) \psi_{m}^{(J)}
$$

Using the set of harmonic oscillator associated with $p_{1}$ or $p_{2}$, one generates a Fock space $\mathscr{F}_{\omega}$ spanned by the states noted $|\omega,\{N\}\rangle$ that are eigenstates of the occupation number operators. The occupation-number-zero state $|\omega, 0\rangle$ is a highest-weight state with weight $\Delta=(C-1) / 24-h \omega^{2} / 4 \pi$. It follows from (2.14) that the operators $\psi$ are naturally defined in a Hilbert space $\mathscr{H}$ of the form:

$$
\mathscr{H} \equiv \bigoplus_{r} \mathscr{F}_{\omega_{r}} ; \quad \omega_{r}=\omega_{0}+r ; \quad r \text { integer. }
$$

Indeed $《 \omega^{\prime},\left\{N^{\prime}\right\}\left|\psi_{m}^{(J)}\right| \omega,\{N\} 》$ is non-vanishing iff $\omega^{\prime}=\omega+2 m$, and $2 m$ is an integer. Physically, the natural choice for $\omega_{0}$ would be the $S L(2, C)$ invariant vacuum (with highest weight zero: $\Delta\left(\omega_{0}\right)=0$ ), that is:

$$
\omega_{0} \equiv \sqrt{\frac{\pi(C-1)}{6 h}}=1+\frac{\pi}{h} .
$$

The mathematical structure we are discussing does not specify $\omega_{0}$, however; and we give it an arbitrary value following the philosophy mentioned in the introduction.

In (2.13) $J$ determines the conformal weight of $\psi_{m}^{(J)}$ :

$$
\Delta(J)=-J-h J(J+1) / \pi \text {. }
$$

The quadratic term of this last expression coincides with the weights of the $S U(2)_{k}$ WZW model if one lets $h=-\pi /(k+2)$. This directly exhibits a relationship between the present Virasoro chiral conformal family and a deformed WZW theory.

As shown in Appendix A, one may define the operators $\psi_{m}^{(J)},-J \leqq m \leqq J$, such that Eq. (2.5b) is generalized to

$$
\psi_{i}(\sigma) \psi_{m}^{(J)}\left(\sigma^{\prime}\right)=\sum_{j=1,2 ; n=-J \ldots J} S_{i m}^{(J) n j} \psi_{n}^{(J)}\left(\sigma^{\prime}\right) \psi_{j}(\sigma),
$$

where the non-vanishing matrix elements are given by

$$
\begin{gathered}
S_{1 m}^{(J) m 1}(\omega)=S_{2,-m}^{(J)-m, 2}(-\omega)=\frac{\lfloor\omega+J+m\rfloor}{\lfloor\omega\rfloor} e^{i h m \varepsilon}, \\
S_{1, m}^{(J) m-1,2}(\omega)=\frac{\lfloor J+m\rfloor}{\lfloor\omega\rfloor} e^{i h \varepsilon(1-m-\omega)}=S_{2,-m}^{(J)-m+1,1}(\omega) .
\end{gathered}
$$

Finally, it is further shown in Appendix A, that the general fusion rules, to leading order are given by:

$$
\begin{gathered}
\psi_{m}^{(J)} \psi_{m^{\prime}}^{\left(J^{\prime}\right)} \sim \mathbf{N}\left(J, m ; J^{\prime}, m^{\prime} ; \omega\right) \psi_{m+m^{\prime}}^{\left(J+J^{\prime}\right)}, \\
\mathbf{N}=\prod_{r=0}^{J-m-1} \frac{\left\lfloor\omega+J+J^{\prime}+m+m^{\prime}-r\right\rfloor}{\lfloor\omega+J+m-r\rfloor} \prod_{s=0}^{J+m-1} \frac{\left\lfloor\omega-J-J^{\prime}+m+m^{\prime}+s\right\rfloor}{\lfloor\omega-J+m+s\rfloor} .
\end{gathered}
$$

All the consistency conditions of conformal algebras are satisfied, but take an unusual form since $S$ and $\mathbf{N}$ depend upon $\omega$ and thus do not commute with the 
primary fields. As we shall see, the operator product structure just displayed is associated with the quantum group $S L(2)_{q}$. This fact is hidden at this moment and will appear when we choose another basis of conformal primary fields. The present basis has simple shift properties in view of (2.14), and the fields are periodic in $\sigma$ up to a constant. However, this choice is inappropriate from the quantum group viewpoint to be discussed in the next section.

Let us finally discuss the classical limit of this conformal structure in order to make contact with what we recalled in the introduction. We shall let $\gamma$ go to zero. In this limit $h_{-} \approx 2 \pi \gamma$, and, letting $h=h_{-}$gives $\psi_{j} \approx d_{j} e^{\sqrt{\gamma} \phi_{j}}$. If one retains only the - operators, one goes smoothly back to the classical case. One expects that commutators will behave as $i \gamma$ times the Poisson brackets. Looking at (1.13) one sees that this will be true if $p_{n}^{(j)} \approx 1 / \sqrt{\gamma}$. According to (2.6) this means that $h \omega$ will tend to a constant. With this information one immediately deduces from $(2.4,5 \mathrm{a}, 5 \mathrm{~b})$ that

$$
\begin{aligned}
& \psi_{j}(\sigma) \psi_{j}\left(\sigma^{\prime}\right) \approx \psi_{j}\left(\sigma^{\prime}\right) \psi_{j}(\sigma)(1-i \pi \gamma \varepsilon), \\
& \psi_{1}(\sigma) \psi_{2}\left(\sigma^{\prime}\right) \approx \psi_{2}\left(\sigma^{\prime}\right) \psi_{1}(\sigma)(1+i \pi \gamma \varepsilon+2 \pi \gamma \cot (h \omega))+\psi_{1}\left(\sigma^{\prime}\right) \psi_{2}(\sigma)\left(\frac{2 \pi \gamma e^{-i h \omega \varepsilon}}{\sin (2 \pi \gamma \omega)}\right), \\
& \psi_{2}(\sigma) \psi_{1}\left(\sigma^{\prime}\right) \approx \psi_{1}\left(\sigma^{\prime}\right) \psi_{2}(\sigma)(1+i \pi \gamma \varepsilon-2 \pi \gamma \cot (h \omega))-\psi_{2}\left(\sigma^{\prime}\right) \psi_{1}(\sigma)\left(\frac{2 \pi \gamma e^{-i h \omega \varepsilon}}{\sin (2 \pi \gamma \omega)}\right) .
\end{aligned}
$$

This immediately leads to the PB exchange Eq. (1.14).

\section{Connection with the Universal $R$ Matrix}

Introduce

with

$$
\xi_{\alpha}=\sum_{i=1,2} u_{\alpha}^{i} \psi_{i} \text { for } \alpha=1,2
$$

$$
u_{1}^{1}=u_{2}^{2}=e^{i h \omega / 2} ; \quad u_{2}^{1}=e^{-i h(\omega+1) / 2} ; \quad u_{1}^{2}=e^{-i h(\omega-1) / 2} .
$$

After some computation, one deduces [3] from $(2.4,5)$ that the $\xi$ fields so defined satisfy the exchange algebra

$$
\xi_{\alpha}(\sigma) \xi_{\beta}\left(\sigma^{\prime}\right)=\sum_{\gamma, \delta} \rho_{\alpha \beta}^{\gamma \delta} \xi_{\gamma}\left(\sigma^{\prime}\right) \xi_{\delta}(\sigma),
$$

where, for $\varepsilon=1$, the only non-vanishing matrix elements are

$$
\begin{gathered}
\rho_{11}^{11}=\rho_{22}^{22}=e^{-i h / 2}, \\
\rho_{12}^{21}=\rho_{21}^{12}=e^{i h / 2} ; \quad \rho_{21}^{21}=e^{-i h / 2}-e^{3 i h / 2} .
\end{gathered}
$$

This last expression coincides with the simplest $R$ matrix of $S L(2)_{q}$ with $q=e^{-i h / 2}$. It corresponds to $J=1 / 2$ in the above family of primary fields, and our task is to generalize this result to arbitrary $J$. Derivations will be sketched below. Details are given in Appendix B, C and D. The general formulae are as follows. Let

$$
\left.\xi_{M}^{(J)}(\sigma) \equiv \sum_{-J \leqq m \leqq J} \mid J, \omega\right)_{M}^{m} \psi_{m}^{(J)}(\sigma)
$$


where the coefficients $\mid J, \omega)_{M}^{m}$ are given by:

$$
\begin{aligned}
& \mid J, \omega)_{M}^{m}=\left(e^{-i h \frac{a_{2}}{a_{1}}}\right)^{J+M} \kappa_{J} \sqrt{\left(\begin{array}{c}
2 J \\
J+M
\end{array}\right)} e^{i h m / 2} \\
& \text {. } \sum_{(J-M+m-s) / 2 \text { integer }} e^{i h s(\omega+m)}\left(\begin{array}{c}
J-M \\
(J-M+m-s) / 2
\end{array}\right)\left(\begin{array}{c}
J+M \\
(J+M+m+s) / 2
\end{array}\right) \text {. }
\end{aligned}
$$

The constants $a_{i}$, and $\kappa_{J}$ depend upon the normalizations of the $\xi$ fields. In particular, the $a_{i}$ 's are such that $\xi_{-1 / 2}^{(1 / 2)} \equiv a_{1} \xi_{1}, \xi_{1 / 2}^{(1 / 2)} \equiv a_{2} \xi_{2}$, if we let $\kappa_{1 / 2}=a_{1}$. The $q$-deformed binomial coefficients have been introduced by the formula:

where

$$
\left(\begin{array}{l}
P \\
Q
\end{array}\right) \equiv \frac{\lfloor P\rfloor !}{\lfloor Q\rfloor !\lfloor P-Q\rfloor !},
$$

$$
\lfloor n\rfloor ! \equiv \prod_{r=1}^{n}[r]
$$

The operators so defined satisfy the general exchange algebra

$$
\xi_{M}^{(J)}(\sigma) \xi_{M^{\prime}}^{\left(J^{\prime}\right)}\left(\sigma^{\prime}\right)=\sum_{-J \leqq N \leqq J ;-J^{\prime} \leqq N^{\prime} \leqq J^{\prime}}\left(J, J^{\prime}\right)_{M M^{\prime}}^{N^{\prime} N} \xi_{N^{\prime}}^{\left(J^{\prime}\right)}\left(\sigma^{\prime}\right) \xi_{N}^{(J)}(\sigma) .
$$

The coefficients $\left(J, J^{\prime}\right)_{M M^{\prime}}^{N^{\prime} N}$ of the $R$-matrix only depend upon the sign of $\sigma-\sigma^{\prime}$. This matrix is conveniently written in terms of quantum group generators as follows: Introduce a set of Hilbert spaces $H_{J}$ with states notes $|J, M\rangle,-J \leqq M \leqq J$; together with operators $J_{ \pm}, J_{3}$ such that:

$$
J_{ \pm}|J, M\rangle=\sqrt{\lfloor J \mp M\rfloor\lfloor J \pm M+1\rfloor}|J, M \pm 1\rangle J_{3}|J, M\rangle=M|J, M\rangle .
$$

These operators satisfy the $S L(2)_{q}$ commutation relation

For $\sigma>\sigma^{\prime}$, one has:

$$
\left[J_{+}, J_{-}\right]=\left\lfloor 2 J_{3}\right\rfloor \text {. }
$$

$$
\begin{gathered}
\left(J, J^{\prime}\right)_{M M^{\prime}}^{N^{\prime} N}=\left(\left\langleJ, M\left|\otimes\left\langle J^{\prime}, M^{\prime}\right|\right) \mathbf{R}\left(|J, N\rangle \otimes\left|J^{\prime}, N^{\prime}\right\rangle\right),\right.\right. \\
\mathbf{R}=e^{\left(-2 i h J_{3} \otimes J_{3}\right)}\left(1+\sum_{n=1}^{\infty} \frac{\left(1-e^{2 i h}\right)^{n} e^{i h n(n-1) / 2}}{\lfloor n\rfloor !} e^{-i h n J_{3}}\left(J_{+}\right)^{n} \otimes e^{i h n J_{3}}\left(J_{-}\right)^{n}\right) .
\end{gathered}
$$

The last formula exactly reproduces the universal $R$ matrix of $S L(2)_{q}[1]$ with $q=e^{-i h / 2}$. The case $\sigma<\sigma^{\prime}$ will be discussed below.

Moreover, to leading order in the singularity, at $\sigma \rightarrow \sigma^{\prime}$, the fusion rules are given by:

$$
\begin{gathered}
\xi_{M}^{(J)}(\sigma) \xi_{M^{\prime}}^{\left(J^{\prime}\right)}\left(\sigma^{\prime}\right) \sim\left(1-e^{-i\left(\sigma-\sigma^{\prime}\right)}\right)^{-2 h J J^{\prime} / \pi} \lambda\left(J, M ; J^{\prime}, M^{\prime}\right) \xi_{M+M^{\prime}}^{\left(J+J^{\prime}\right)}(\sigma), \\
\lambda\left(J, M ; J^{\prime}, M^{\prime}\right)=\frac{\kappa_{J} \kappa_{J^{\prime}}}{\kappa_{J+J^{\prime}}} \sqrt{\frac{\left(\begin{array}{c}
2 J \\
J+M
\end{array}\right)\left(\begin{array}{c}
2 J^{\prime} \\
J^{\prime}+M^{\prime}
\end{array}\right)}{\left(\begin{array}{c}
2 J+2 J^{\prime} \\
J+J^{\prime}+M+M^{\prime}
\end{array}\right)}} e^{i h\left(M^{\prime} J-M J^{\prime}\right)},
\end{gathered}
$$


Proof. One proceeds by recursion starting from (3.1). Some details are given in Appendix B, C and D. The basic requirement that determines the coefficients $\mid J, \omega)_{M}^{m}$ of (3.3) is that all $\omega$ dependence disappear from the exchange algebra and from the fusion rules. In Appendix B, Eq. (3.4) is established by studying the exchange of $\xi^{(1 / 2)}$ with $\xi^{(J)}$ for arbitrary $J$. In Appendix $C$, the coefficients $\lambda(1 / 2, \pm 1 / 2 ; J, M)$ are first explicitly computed using the results of Appendix A. Equation (3.13) is then established from the associativity of the short distance operator product expansion. Finally, in Appendix D, the general exchange algebra is derived from the requirement that the order between fusion and exchange be irrelevant. This leads to recurrence formulae which are solved by first taking the expression (3.11) with arbitrary coefficients as an ansatz, and showing that the universal $R$-matrix is the solution. For this, a basic relation is

$$
\left(J_{ \pm}\right)^{p}|J, M\rangle=\sqrt{\frac{\lfloor J \mp M\rfloor !\lfloor J \pm M+p\rfloor}{\lfloor J \mp M-p] !\lfloor J \pm M\rfloor !}}|J, M \pm p\rangle
$$

that easily follows from (3.8). Q.E.D.

Our next topic is the hermiticity relation of the conformal family we just discussed. We shall assume at this point that $h$ is real. On Eq. (2.3) one sees that this is true for $C>25(0<\gamma<1 / 8)$ which is the weak coupling regime of $2 \mathrm{D}$ gravity and for $C<1(\gamma<0)$ which is the standard regime of minimal models. In this case $\omega$ is real and Eq. (2.6) shows that $p_{0}^{(1)}=-p_{0}^{(2)}$ are pure imaginary ${ }^{4}$. Thus

$$
\left(p_{0}^{(1)}\right)^{\dagger}=p_{0}^{(2)} \text {. }
$$

Neveu and I long ago argued [12] that the correct hermiticity relation is:

$$
p_{1}^{\dagger}=p_{2} \text {. }
$$

Indeed, it is compatible with (3.15) contrary to the standard hermiticity condition for free chiral bosonic fields. Moreover Eq. (2.1) shows that the Virasoro generators have two equivalent expressions:

$$
L_{m}=\int_{0}^{2 \pi} \frac{d \sigma}{4 \pi} N^{(1)}\left(p_{1}^{2}+p_{1}^{\prime} / \sqrt{\gamma}\right)+\frac{1}{8 \gamma} \delta_{m, 0}=\int_{0}^{2 \pi} \frac{d \sigma}{4 \pi} N^{(2)}\left(p_{2}^{2}+p_{2}^{\prime} / \sqrt{\gamma}\right)+\frac{1}{8 \gamma} \delta_{m, 0}
$$

which are interchanged by (3.16) so that one correctly obtains the hermiticity relation $L_{m}^{\dagger}=L_{-m}$. As recalled in the introduction, $p_{1}$ and $p_{2}$ are related by a generalized Galois transformation. It is a standard phenomenon that complex conjugation may interchange solutions of an algebraic equation with real coefficients and may therefore correspond to a Galois transformation. We now show that the exchange algebras we have derived have simple transformation properties under (3.16). The basic point is to recall that the fields $\psi_{m}^{(J)}$ do not

\footnotetext{
${ }^{4}$ For simplicity, I only consider the case $0<\gamma<1 / 8$ where $h>0$. The case $\gamma<0$ is treated similarly
} [12]. There are some extra minus signs. In particular (3.16) is replaced by $p_{1}^{\dagger}=-p_{2}$ 
commute with $\omega$ as shown on (2.14). Equation (2.2) with $j=1$ gives

$$
\begin{aligned}
\psi_{1}^{\dagger} & =N^{(2)}\left(e^{\sqrt{h / 2 \pi} \phi_{2}}\right) d_{1}(\omega)^{\dagger}=d_{1}(\omega+1)^{\dagger} N^{(2)}\left(e^{\sqrt{h / 2 \pi} \phi_{2}}\right) \\
& =\frac{d_{1}(\omega+1)^{\dagger} d_{2}(\omega)^{\dagger}}{d_{2}(\omega) d_{2}(\omega)^{\dagger}} \psi_{2}=\frac{\Gamma(-(\omega+1) h / \pi) \Gamma(\omega h / \pi)}{\left|d_{2}(\omega)\right|^{2}} \psi_{2} .
\end{aligned}
$$

The last factor is real but not always positive. The general discussion may be carried out if needed, but would be cumbersome. Here we only consider the values of $\omega$ where this factor is positive ${ }^{5}$. The we may choose the $d_{i}$ 's to be real and $d_{1}(\omega+1)=d_{2}(\omega)$, obtaining $\psi_{1}^{\dagger}=\psi_{2}$. Equation (2.5a) becomes:

$$
d_{1}(\omega)=d_{2}(\omega-1)=\sqrt{\Gamma(-\omega h / \pi) \Gamma((\omega-1) h / \pi)}
$$

which also gives $\psi_{2}^{\dagger}=\psi_{1}$ : the square of the complex conjugation is indeed the identity. It is shown, in Appendix A that this extends to the higher $\psi$ operators, as

$$
\left(\psi_{m}^{(J)}\right)^{\dagger}=\psi_{-m}^{(J)} .
$$

Concerning the $\xi$-operators, Eq. (3.3) leads to

$$
\left.\left.\xi_{M}^{(J)}(\sigma)^{\dagger}=\sum_{m} \psi_{-m}^{(J)}(\sigma)(\mid J, \omega)_{M}^{m}\right)^{\dagger}=\sum_{m}(\mid J, \omega+2 m)_{M}^{-m}\right)^{\dagger} \psi_{m}^{(J)}(\sigma) .
$$

Using the fact that $\left(\begin{array}{l}P \\ Q\end{array}\right)=\left(\begin{array}{c}P \\ P-Q\end{array}\right)$, it is easy to verify from (3.4) that, if we choose, $a_{2} / a_{1}=e^{i h}$ and $\kappa_{J}$ real, we have

so that

$$
\left.\left.(\mid J, \omega+2 m)_{M}^{-m}\right)^{\dagger}=\mid J, \omega\right)_{M}^{m}
$$

$$
\xi_{M}^{(J)}(\sigma)^{\dagger}=\xi_{M}^{(J)}(\sigma)
$$

are hermitian fields. Concerning the exchange algebras, one may verify that the scheme is completely consistent with the present hermiticity structure. Consider in particular the hermitian conjugate of $(3.7,10)$. One obtains

$$
\xi_{M^{\prime}}^{\left(J^{\prime}\right)}\left(\sigma^{\prime}\right) \xi_{M}^{(J)}(\sigma)=\sum_{-J \leqq N \leqq J ;-J^{\prime} \leqq N^{\prime} \leqq J^{\prime}}\left(\left(J, J^{\prime}\right)_{M M^{\prime}}^{N^{\prime} N}\right)^{*} \xi_{N^{\prime}}^{\left(J^{\prime}\right)}\left(\sigma^{\prime}\right) \xi_{N}^{(J)}(\sigma),
$$

where $\left(\left(J, J^{\prime}\right)_{M M^{\prime}}^{N^{\prime} N}\right)^{*}$ is the complex conjugate of $\left(J, J^{\prime}\right)_{M M^{\prime}}^{N^{\prime} N}$ In (3.7) the operators on the left-hand side are ordered with decreasing arguments, while the opposite is true in (3.24). The latter equation thus corresponds to the other $R$-matrix which has been left out of the discussion so far. For consistency, it is convenient to define

$$
\overline{\left(J^{\prime}, J\right)_{M^{\prime} M}^{N}} \equiv\left(\left(J, J^{\prime}\right)_{M}^{N^{\prime} M^{\prime}}\right)^{*}
$$

so that

$$
\xi_{M^{\prime}}^{\left(J^{\prime}\right)}\left(\sigma^{\prime}\right) \xi_{M}^{(J)}(\sigma)=\sum_{-J \leqq N \leqq J ;-J^{\prime} \leqq N^{\prime} \leqq J^{\prime}} \overline{\left(J^{\prime}, J\right)_{M^{\prime} M}^{N N^{\prime}}} \xi_{N}^{(J)}(\sigma) \xi_{N^{\prime}}^{\left(J^{\prime}\right)}\left(\sigma^{\prime}\right)
$$

5 This is true for instance if $\omega>0$ and $(2 q+2) \pi / h>\omega+1>(2 q+1) \pi / h, q$ positive integer. The width of this interval is $\pi / h$ and thus involves several values of $\omega$ of the form $(2.15 a)$ iff $\pi / h>1$. This last condition holds if we choose $h=h_{-}$ 
has the same form as (3.7). It may also be written under a form similar to $(3.10,11)$. Using the fact that the matrix elements of $J_{ \pm}$and $J_{3}$ are real (see (3.8)), one easily derives

$$
\begin{aligned}
& \overline{\left(J^{\prime}, J\right)_{M^{\prime} M}^{N N^{\prime}}}=\left(\left\langleJ^{\prime}, M^{\prime}|\otimes\langle J, M|) \overline{\mathbf{R}}\left(\left|J^{\prime}, N^{\prime}\right\rangle \otimes|J, N\rangle\right),\right.\right. \\
& \overline{\mathbf{R}}=e^{\left(2 i h J_{3} \otimes J_{3}\right)}\left(1+\sum_{n=1}^{\infty} \frac{\left(1-e^{-2 i h}\right)^{n} e^{-i h n(n-1) / 2}}{\lfloor n\rfloor !} e^{-i h n J_{3}}\left(J_{-}\right)^{n} \otimes e^{i h n J_{3}}\left(J_{+}\right)^{n}\right) .
\end{aligned}
$$

Applying (3.7) and (3.24) in succession must give the identity. This gives the inverse relation:

$$
\sum_{-J \leqq N \leqq J ;-J^{\prime} \leqq N^{\prime} \leqq J^{\prime}}\left(J, J^{\prime}\right)_{M M^{\prime}}^{N^{\prime} N} \overline{\left(J^{\prime}, J\right)_{N^{\prime} N}^{P P^{\prime}}}=\delta_{M, P} \delta_{M^{\prime}, P^{\prime}},
$$

whose proof is based on the interesting identity:

$$
\sum_{n+m=N ; n \geqq 0, m \geqq 0}(-1)^{m} \frac{e^{i n(n-m)(1-N) / 2}}{[n] !\lfloor m\rfloor !}=0, \quad \text { if } \quad N>0 .
$$

This latter equation is the $q$-deformation of a standard identity on binomial coefficients. Its actual derivation is left to the dedicated reader.

As a general remark let us note that the hermiticity condition discussed here is the quantum equivalent of the allowed region for the classical Schrödinger equation (1.6). Indeed, for oscillatory waves the eigenvalues of the monodromy matrix are complex conjugate and of modulus one. The two Bloch waves are complex conjugate, which is the classical analogue of (3.20).

For the forbidden region of the classical Schrödinger equation, the Bloch waves are real. Quantum mechanically this corresponds to $p_{j}^{\dagger}=p_{j}$. This other hermiticity relation is relevant for $1 / 8<\gamma<\infty[5,12,13]$, where $h_{ \pm}$are complex and $h_{-}=h_{+}^{*}$. Thus both values of $h$ must be retained. This is the subject of the coming section.

\section{The Complete Algebra}

Let us finally derive the complete algebra by taking both values of $h$ in (2.3) into account. The notation of the above section must be doubled. It will be simplest to still denote by $h$ one of the two choices (for instance $h_{-}$), and to write all symbols related to it as in the previous sections. The other value (for instance $h_{+}$) will be denoted by $\hat{h}$ and its associated symbols distinguished by a hat. This avoids clumsy notation, at the expense of the explicit symmetry between $h$ and $\hat{h}$. One now has:

$$
\begin{aligned}
& h \hat{h}=\pi^{2} ; \quad q=e^{-i h / 2}, \quad \hat{q}=e^{-i \hat{h} / 2}, \\
& \omega=i p_{0}^{(1)} \sqrt{\frac{2 \pi}{h}} ; \quad \hat{\omega}=i p_{0}^{(1)} \sqrt{\frac{2 \pi}{\hat{h}}} ; \quad \hat{\omega}=\omega \frac{h}{\pi} ; \quad \omega=\hat{\omega} \frac{\hat{h}}{\pi} \\
& \hat{\psi}_{i} \equiv \hat{d}_{i} N^{(i)}\left(e^{\sqrt{h / 2 \pi} \phi_{i}}\right)=\hat{d}_{i} N^{(i)}\left(e^{(\pi / h) \sqrt{h / 2 \pi} \phi_{i}}\right) .
\end{aligned}
$$


Some details are given in Appendix E. One arrives at a general family $\psi_{m \hat{m}}^{(\hat{J})},-J \leqq m \leqq J,-\hat{J} \leqq \hat{m} \leqq \hat{J}$. The fusion to leading order is given by:

$$
\begin{aligned}
& \psi_{m \hat{m}}^{(J \hat{J})}(\sigma) \psi_{m^{\prime} \hat{m}^{\prime}}^{\left(J^{\prime}\right)}\left(\sigma^{\prime}\right) \rightarrow\left(1-e^{-i\left(\sigma-\sigma^{\prime}\right)}\right)^{\Delta_{\mathrm{Kac}}\left(J+J^{\prime}, \hat{J}+\hat{J}^{\prime} ; C\right)-\Delta_{\mathrm{Kac}}(J, \hat{J} ; C)-\Delta_{\mathrm{Kac}}\left(J^{\prime}, \hat{J}^{\prime} ; C\right)} \\
& \cdot \mathbf{N}\left(J, m, \hat{J}, \hat{m} ; J^{\prime}, m^{\prime}, \hat{J}^{\prime}, \hat{m}^{\prime} ; \omega\right) \psi_{m+m^{\prime} \hat{m}^{\prime}+\hat{m}^{\prime}}^{\left(J+\hat{J}^{\prime}\right.}(\sigma),
\end{aligned}
$$

where

$$
\begin{aligned}
\mathbf{N}\left(J, m, \hat{J}, \hat{m} ; J^{\prime}, m^{\prime}, \hat{J}^{\prime}, \hat{m}^{\prime} ; \omega\right)= & (-1)^{2\left(J \hat{J}^{\prime}+\hat{J} J^{\prime}-m \hat{m}^{\prime}-\hat{m} m^{\prime}\right)} \\
& \cdot \mathbf{N}\left(J, m ; J^{\prime}, m^{\prime} ; \omega\right) \hat{\mathbf{N}}\left(\hat{J}, \hat{m} ; \hat{J}^{\prime}, \hat{m}^{\prime} ; \hat{\omega}\right) .
\end{aligned}
$$

The divergent factor shows that the conformal weights take the form $\Delta=\Delta_{\mathrm{Kac}}(J, \widehat{J} ; C)$, where $\Delta_{\mathrm{Kac}}$ is given by Kac's formula: ${ }^{6}$

$$
\Delta_{\mathrm{Kac}}(J, \hat{J} ; C)=\frac{C-1}{24}-\frac{1}{24}((J+\hat{J}+1) \sqrt{C-1}-(J-\hat{J}) \sqrt{C-25})^{2} .
$$

In terms of the WZW $\Delta(J)$ given by (2.16), this may be written as

$$
\Delta_{\mathrm{Kac}}(J, \hat{J} ; C) \equiv \Delta\left(J+\frac{\pi}{h} \hat{J}\right)=\hat{\Delta}\left(\hat{J}+\frac{h}{\pi} J\right),
$$

which reflects the fact that, formally, $\psi_{m \hat{m}}^{(J \hat{J})} \approx \psi_{(m+\hat{m}(\pi / h)) 0}^{((J+\hat{\jmath}(\pi / h))}$, and $\psi_{m \hat{m}}^{(J \hat{J})} \approx \psi_{0(m(h / \pi)+\hat{m}))}^{(0(J(h / \pi)+\hat{j})}$. Although formal since for general $h$ neither $\pi / h$ nor $h / \pi$ are integers, this connection is of help in deriving the properties of the $\psi_{m \hat{m}}^{(\hat{J})}$ as shown in Appendix E. Equation (4.4) shows, in particular that

$$
\psi_{m 0}^{(J 0)} \psi_{0 \hat{m}}^{(0 \hat{J})} \sim \psi_{0 \hat{m}}^{(0 \hat{J})} \psi_{m 0}^{(J 0)} \sim(-1)^{2(J \hat{J}-m \hat{m})} \psi_{m \hat{m}}^{(J \hat{J})} .
$$

The appropriate definition of the $\xi$ fields is

$$
\left.\left.\xi_{M \hat{M}}^{(J \hat{J})}(\sigma)=\sum_{-J \leqq m \leqq J ;-\hat{J} \leqq \hat{m} \leqq \hat{J}}(-1)^{4 J \hat{J}} e^{-i \pi(M \hat{J}+\hat{M} J)} \mid J, \omega\right)_{M}^{m} \mid \widehat{J}, \hat{\omega}\right)_{\hat{M}}^{\hat{m}} \psi_{m \hat{m}}^{(J \hat{J})}(\sigma) .
$$

The phase factor $e^{-i \pi(M \hat{J}+\hat{M} J)}$ is such that, under the hermitian structure discussed in Sect. 3,

$$
\xi_{M \hat{M}}^{(J \hat{J})}(\sigma)=\left(\xi_{M \hat{M}}^{(J \hat{J})}(\sigma)\right)^{\dagger}
$$

are again hermitian fields. From (4.8) and (4.9) one deduces that

$$
\begin{aligned}
& \xi_{M}^{(J)} \hat{\xi}_{\hat{M}}^{(\hat{J})} \sim e^{i \pi(M \hat{J}-\hat{M} J)} \xi_{M \hat{M}}^{(J \hat{J})}, \\
& \hat{\xi}_{\hat{M}}^{(\hat{J})} \xi_{M}^{(J)} \sim e^{i \pi(\hat{M} J-M \hat{J})} \xi_{M \hat{M}}^{(J \hat{J})} .
\end{aligned}
$$

On the other hand, one finds that due to the relation (4.1), the $\psi$ and $\hat{\psi}$ fields have a trivial exchange algebra:

$$
\psi_{m 0}^{(J 0)}(\sigma) \psi_{0 \hat{m}}^{(0 \hat{\jmath})}\left(\sigma^{\prime}\right)=e^{-2 i \pi J \hat{J}_{\varepsilon}} \psi_{0 \hat{m}}^{(0 \hat{J})}\left(\sigma^{\prime}\right) \psi_{m 0}^{(J 0)}(\sigma),
$$

which leads to

$$
\xi_{M}^{(J)}(\sigma) \hat{\xi}_{\hat{M}}^{(\hat{J})}\left(\sigma^{\prime}\right)=e^{-2 i \pi J \hat{J}_{\varepsilon}} e^{2 i \pi(M \hat{J}-\hat{M} J)} \hat{\xi}_{\hat{M}}^{(\hat{J})}\left(\sigma^{\prime}\right) \xi_{M}^{(J)}(\sigma)
$$

${ }^{6}$ This particular form was first written in ref. [11] 
in agreement with $(4.11 \mathrm{a}, \mathrm{b})$. Altogether, the properties of the complete family are simple extensions of the ones derived in the previous section: making use of $(4.11 \mathrm{a}, \mathrm{b})$ and (4.13) one sees that the exchange algebra and the fusion coefficients are, up to simple phase coefficients which are easily worked out, products of two copies of the expressions worked out in Sect. 3, one with $h$, and the other with $\hat{h}$. It is, moreover, straightforward to discuss the hermiticity structure recalled at the end of the previous section which corresponds to the classically forbidden zones. I shall not do so here, since the method is very similar to one followed in Sect. 3.

\section{Conclusion}

We have thoroughly established the connection between $S L(2)_{q}$ and a chiral conformal family on the unit circle which one may call universal since its exchange algebra is given by the universal $R$-matrix. Collecting (3.4) (or (B.21)) with (A.14) and (A.13), one sees that

$$
\begin{aligned}
\left\langle\omega_{2}, 0\left|\xi_{M}^{(J)}(\sigma)\right| \omega_{1}, 0 》=\right. & e^{i \sigma\left(\omega_{1}^{2}-\omega_{2}^{2}\right) h / 4 \pi} \\
& \left.\cdot \sum_{-J \leqq m \leqq J} \mid J, \omega_{2}\right)_{M}^{m}\left(C^{J-m, J+m} D^{J-m, J+m}\left(\omega_{2}\right) \delta_{\omega_{2}-\omega_{1}-2 m}\right) .
\end{aligned}
$$

The last $\delta$-function selects out only one term (the coefficient will be non-zero only for a finite number of $\omega$ shifts), and this completely determines the matrix elements of the $\xi$-fields between the highest weight states, that is all three-point couplings. Such is a practical meaning of the quantities $\mid J, \omega)_{M}^{m}, C^{J-m, J+m}, D^{J-m, J+m}$, which we have computed. In the generic case considered, the Verma modules are trivial and from the covariance of the $\xi$-fields one deduces any matrix elements by applying appropriate differential operators to (5.1). Moreover, since we have been working with operators, we may go to the case of a higher genus by taking an appropriate trace. For instance, the torus is obviously dealt with by considering, typically, $\operatorname{Trace}\left(\xi_{M}^{(J)}(\sigma) e^{\tau L_{0}}\right)$.

Another point is that the $N$-point functions of the $V^{\mu, v}$ 's are given by the Coulomb gas picture [10]. For the $\xi$-fields one finds suitable linear combinations of them that are determined from the explicit expressions of $\mid J, \omega)_{M}^{m}, C^{J-m, J+m}$, $D^{J-m, J+m}$.

Physically, the $\psi$ and $\hat{\psi}$ fields are the basic chiral operators which allow us to construct the conformal theories associated to $A_{1}$ in the Toda classification [5]. On the one hand, for $C>1$, they naturally appear in the quantum solution of the Liouville theory [2-5] which is the Toda theory associated with the simple algebra $A_{1}$. On the other hand, for $C<1$ they are the basic tools to recover the $\left(S U(2)_{k} \otimes S U(2)_{1}\right) / S U(2)_{k+1}$ coset models [5].

The structure we have displayed is consistent with the quantum the group realization:

$$
J_{ \pm} \xi_{M}^{(J)}=\sqrt{\lfloor J \mp M\rfloor\lfloor J \pm M+1\rfloor} \xi_{M \pm 1}^{(J)}, \quad J_{3} \xi_{M}^{(J)}=M \xi_{M}^{(J)} .
$$

This precisely defines the quantum group structure that underlies $2 \mathrm{D}$ gravity and 
minimal models. As already indicated, the symmetry between $\psi_{1}$ and $\psi_{2}$ is the basis of the action of the quantum group generators. At this point a comment on the connection between the present work and other related studies is in order. The braiding matrix (2.5) was rediscovered in a recent article [8] which already contains a discussion similar to the one carried out in Sect. 2 and Appendix A: a recursion on the $R$-matrices based on the construction of the conformal families given by fusion of the elementary $(1,2)$ and $(2,1)$ fields. Furthermore, a connection with quantum group has already been put forward in the literature and appears in particular in ref. [8]. This article however only works with the equivalent of our $\psi$-fields. It is important to stress that the viewpoint taken here on quantum groups is different and that the main results of the present article are really new. Indeed the connection between conformal theories and quantum group has been made so far by considering the $q-6-j$ symbols. It is clear that they provide solutions of Yang and Baxter's equation and are thus related to braiding matrices; however, this connection is indirect and does not show how the fields transform under the quantum group action. In the present paper, on the contrary, the $\xi$ fields are constructed in such a way that their exchange algebra coincides with the universal $R$-matrix, and their OPE algebra is thus precisely connected with the standard coproduct of $S L(2)_{q}$, if the action of the generators is given by (5.2). In other words, the $\xi$ fields are such that the true quantum mechanical effects, that is their non-commutativity as quantum operators in the Hilbert space of states, coincides with the non-commutativity induced by the deformation from $S L(2)$ to $S L(2)_{q}$. The latter may thus be regarded as dictated by the former, and the present article takes a basic step in establishing the connection between quantum group and conformal models following a path initiated in ref. [3].

One of the forthcoming problems is to particularize this structure to rational theories. For $C<1$ and $C>25$, this involves specializing $q$ to be a root of unity. Indeed, if $C=1-6\left(p-p^{\prime}\right)^{2} /\left(p p^{\prime}\right)$, one has $h=-\pi p / p^{\prime}, q=\exp \left(i \pi p /\left(2 p^{\prime}\right)\right)$. According to (2.7), $\lfloor x\rfloor$ vanishes if $x$ is a multiple of $p^{\prime}$. Clearly, the results of the present paper do not blindly apply since denominators vanish in many places, such as, for instance, in formula (3.11). Further work is needed. However, for generic $h$, the operator product properties of the $\xi$ fields are entirely given by group theoretic expressions. In particular, although we only determined one type of fusion coefficient, it is clear that they are all given by the $q$ deformed Clebsch Gordon coefficients. Thus, the problem of specializing $q$ to a root of unity has been essentially reduced to the equivalent limit in the representation theory of quantum groups. It is well known that the $q$-Clebsch-Gordon and $q-6 j$ symbols become singular unless the allowed values of $J$ are restricted to $J<p^{\prime} / 2$. In general, the limit $h \rightarrow-\pi p / p^{\prime}$ will be well defined in the corresponding truncated family. (This phenomenon was recently discussed in connection with the relationship between sine-Gordon theory and minimal model [14].) As a starting point, one may verify that, for $p>0, p^{\prime}>0$, formula (3.14) makes sense if one restricts $J$ to $J \leqq P^{\prime} / 2$ and that $J_{ \pm}^{v}|J, M\rangle=0$ with $v=p^{\prime}-J \mp M$. It should be straightforward to work out this truncation in practice, and this should in particular reproduce the spectrum of primary fields of minimal models. I leave this for a later publication since the present article is already rather lengthy. 
For $1<C<25 h$ and $\hat{h}$ are complex conjugate. This is the strong coupling regime of gravity in the conformal gauge. The special values which I derived with Neveu [13], that is, $C=7,13,19$, lead to

$$
h+\hat{h} \equiv \frac{\pi(C-13)}{6}=-\pi, 0, \pi .
$$

Since $q=e^{-i n / 2}$, one sees that $\sqrt{q \hat{q}}$ is a fourth root of unity. This is the strong coupling equivalent of the rational case, and the introduction of the $\xi$ and $\hat{\xi}$ fields sheds a new light on this difficult problem [15].

\section{Appendix A}

In this appendix, we establish the properties of the family of $\psi$ operators which will be connected with the quantum group $S L(2)_{q}$ in the following. The precise definition of $\psi_{i}$ is:

where

$$
\psi_{j} \equiv d_{j}(\omega) V_{j}
$$

$$
\begin{aligned}
V_{j} \equiv & N^{(j)}\left(e^{\sqrt{h / 2 \pi} \phi_{j}}\right) \equiv e^{\sqrt{h / 2 \pi} q_{0}^{(j)}} e^{-i \sigma( \pm \omega+1 / 2) h / 2 \pi} \\
& \cdot \exp \left(\sqrt{h / 2 \pi} \sum_{n<0} \frac{p_{n}^{(j)}}{n} e^{-i n \sigma}\right) \exp \left(\sqrt{h / 2 \pi} \sum_{n>0} \frac{p_{n}^{(i)}}{n} e^{-i n \sigma}\right),
\end{aligned}
$$

where the \pm is + for $V_{1}$ and - for $V_{2}$. The definition of primary fields is appropriate the unit circle: $\left[L_{n}, A_{\Delta}\right]=-i e^{i n \sigma}(d / d \sigma+i n \Delta) A_{\Delta}$. As usual, $e^{\sqrt{h / 2 \pi} q_{0}^{(j)}}$ is a formal exponential to be understood as a shift operator. It is such that:

$$
e^{\sqrt{h / 2 \pi} q_{0}^{(i)}} f(\omega)=f\left(\omega+\varepsilon_{i}\right) e^{\sqrt{h / 2 \pi} q_{0}^{(i)}} \quad \varepsilon_{2}=-\varepsilon_{1}=1
$$

for any function $f(\omega)$. Similarly one defines any powers of $V_{i}$ as

$$
V_{j}^{\mu} \equiv N^{(j)}\left(e^{\mu \sqrt{h / 2 \pi} \phi_{j}}\right) ; \mu \text { integer }
$$

with conformal weight

$$
\Delta(\mu)=\frac{-1}{2}\left(\mu+\frac{h}{2 \pi} \mu(\mu+2)\right) .
$$

From the differential equation satisfied by the $V_{j}$ 's one deduces [2]

$$
\begin{aligned}
& 《 \omega_{2}, 0\left|V_{i}(\sigma) A_{\Delta}\left(\sigma^{\prime}\right)\right| \omega_{1}, 0 》=e^{i \sigma^{\prime}\left(\omega_{1}^{2}-\omega_{2}^{2}\right) h / 4 \pi} e^{i\left(\sigma-\sigma^{\prime}\right)\left(-1 / 2 \pm \omega_{f}\right) h / 2 \pi} \\
& \cdot\left(1-e^{-i\left(\sigma-\sigma^{\prime}\right)}\right)^{\beta} F\left(a, b ; c ; e^{-i\left(\sigma-\sigma^{\prime}\right)}\right) \text {, } \\
& a=\beta-\frac{h}{\pi} \pm h\left(\frac{\omega_{2}-\omega_{1}}{2 \pi}\right) ; \quad b=\beta-\frac{h}{\pi} \pm h\left(\frac{\omega_{2}+\omega_{1}}{2 \pi}\right) ; \quad c=1 \pm \frac{h \omega_{2}}{\pi} ; \\
& \beta=\frac{1}{2}(1+h / \pi)\left(1-\sqrt{1-\frac{8 h \Delta}{2 \pi(1+h / \pi)^{2}}}\right),
\end{aligned}
$$

where $F(a, b ; c ; x)$ is the standard hypergeometric function, and $|\omega, 0\rangle$ denotes the 
highest weight states introduced in Sect. 2. If the weight $\Delta$ is equal to $\Delta(\mu)$ for some $\mu$, one finds:

$$
\beta=-\frac{\mu h}{2 \pi}=\Delta(\mu+1)-(\Delta(\mu)+\Delta(1))
$$

which coincides with the expected singularity of the short distance expansion if $\mu$ is an additive variable. This is elementary for products of $V_{j}$ with $V_{j}^{\mu}$ :

$$
V_{j}(\sigma) V_{j}^{\mu}\left(\sigma^{\prime}\right) \sim\left(1-e^{-i\left(\sigma-\sigma^{\prime}\right)}\right)^{\Delta(\mu+1)-\Delta(\mu)-\Delta(1)} V_{j}^{\mu+1}(\sigma) .
$$

However this is non-trivial for products of $V_{1}$ with $V_{2}^{\mu}$ or $V_{2}$ with $V_{1}^{\mu}$. In general, one finds that, to leading order, the branching rules of the short distance expansion do not depend upon $j$. This reflects the fact that

$$
p_{n}^{(1)} \sim p_{n}^{(2)} \text { for } n \rightarrow \infty \text {. }
$$

In general, one defines a two parameter family of operators $V^{\mu, v}$ such that $V^{\mu, 0} \equiv V_{1}^{\mu}, V^{0, v} \equiv V_{2}^{\mu}$. One may show from (A.6-8) that they are defined recursively from the relations

$$
\begin{aligned}
& V_{1} V^{\mu, v} \sim\left(\frac{\Gamma(1+\omega h / \pi) \Gamma(1+(\mu+v+1) h / \pi)}{\Gamma(1+(\omega+v) h / \pi) \Gamma(1+(\mu+1) h / \pi)}\right) V^{\mu+1, v} \\
& V_{2} V^{\mu, v} \sim\left(\frac{\Gamma(1-\omega h / \pi) \Gamma(1+(\mu+v+1) h / \pi)}{\Gamma(1+(-\omega+v) h / \pi) \Gamma(1+(v+1) h / \pi)}\right) V^{\mu, v+1}
\end{aligned}
$$

where $\sim$ means that one retains the leading order at $\sigma \rightarrow \sigma^{\prime}$, and divides by the factor:

$$
\left(1-e^{-i\left(\sigma-\sigma^{\prime}\right)}\right)^{\Delta(\mu+v+1)-\Delta(\mu+v)-\Delta(1)} .
$$

Thus the weight of $V^{\mu v}$ is

$$
\Delta^{\mu v}=\Delta(\mu+v)
$$

Moreover, the normalization of the operators is specified by the condition

$$
《 \omega_{2}, 0\left|V^{\mu v}(\sigma)\right| \omega_{1}, 0 》=e^{i \sigma\left(\omega_{1}^{2}-\omega_{2}^{2}\right) h / 4 \pi} \delta_{\omega_{2}-\omega_{1}+v-\mu} .
$$

Our next task is to determine the general $\psi$ fields such that all $\Gamma$ functions are replaced by trigonometric functions. One finds:

$$
\begin{gathered}
\psi^{\mu, v} \equiv\left(C^{\mu, v} D^{\mu, v}(\omega)\right) V^{\mu, v}, \\
C^{1,0}=C^{0,1}=1 ; \quad D^{1,0}=d_{1} ; \quad D^{0,1}=d_{2}, \\
d_{1}(\omega) d_{2}(\omega-1)=\Gamma(-\omega h / \pi) \Gamma((\omega-1) h / \pi),
\end{gathered}
$$

where the $C$ coefficients are determined by the relations:

$$
\begin{aligned}
& C^{\mu+1, v}=C^{\mu, v} \frac{\Gamma(1+(\mu+v+1) h / \pi)}{\Gamma(1+(\mu+1) h / \pi)}, \\
& C^{\mu, v+1}=C^{\mu, v} \frac{\Gamma(1+(\mu+v+1) h / \pi)}{\Gamma(1+(v+1) h / \pi)},
\end{aligned}
$$


with solution

$$
C^{\mu, v}=\frac{\prod_{r=1}^{\mu+v} \Gamma(1+r h / \pi)}{\prod_{s=1}^{\mu} \Gamma(1+s h / \pi) \prod_{t=1}^{v} \Gamma(1+t h / \pi)} .
$$

Similarly, the $D$ coefficients satisfy

$$
\begin{aligned}
& D^{\mu+1, v}(\omega)=D^{\mu, v}(\omega-1) \frac{\Gamma(-(\omega+v) h / \pi)}{\Gamma(-\omega h / \pi))} d_{1}(\omega), \\
& D^{\mu, v+1}(\omega)=D^{\mu, v}(\omega+1) \frac{\Gamma((\omega-\mu) h / \pi)}{\Gamma(\omega h / \pi))} d_{2}(\omega) .
\end{aligned}
$$

For $\mu>v$, the solution reads:

$$
\left.\left.D^{\mu, v}(\omega)=\prod_{t=1}^{\mu-v}\left(\frac{d_{1}(\omega-t+1)}{\Gamma((\omega-t) h / \pi)}\right) \prod_{r=1}^{\mu} \Gamma((\omega-r) h / \pi)\right) \prod_{s=1}^{v} \Gamma((-\omega-s) h / \pi)\right) .
$$

For $\mu<v$, it is given by:

$$
\left.\left.D^{\mu, v}(\omega)=\prod_{t=1}^{\nu-\mu}\left(\frac{d_{2}(\omega+t-1)}{\Gamma((-\omega-t) h / \pi)}\right) \prod_{r=1}^{\mu} \Gamma((\omega-r) h / \pi)\right) \prod_{s=1}^{v} \Gamma((-\omega-s) h / \pi)\right) .
$$

Next the fusion coefficients to leading order

satisfy the recursions:

$$
\psi^{\mu, v} \psi^{\mu^{\prime}, v^{\prime}} \sim N\left(\mu, v ; \mu^{\prime} v^{\prime} ; \omega\right) \psi^{\mu+\mu^{\prime}, v+v^{\prime}}
$$

$$
\begin{aligned}
& N\left(\mu+1, v ; \mu^{\prime}, v^{\prime} ; \omega\right)=N\left(\mu, v ; \mu^{\prime}, v^{\prime} ; \omega-1\right) \frac{N\left(1,0 ; \mu+\mu^{\prime}, v+v^{\prime} ; \omega\right)}{N(1,0 ; \mu, v ; \omega)}, \\
& N\left(\mu, v+1 ; \mu^{\prime}, v^{\prime} ; \omega\right)=N\left(\mu, v ; \mu^{\prime}, v^{\prime} ; \omega+1\right) \frac{N\left(0,1 ; \mu+\mu^{\prime}, v+v^{\prime} ; \omega\right)}{N(0,1 ; \mu, v ; \omega)} .
\end{aligned}
$$

An explicit computation based on (A.6) and (2.11) leads to:

$$
N\left(1,0 ; \mu^{\prime} v^{\prime} ; \omega\right)=\frac{\left\lfloor\omega+v^{\prime}\right\rfloor}{\lfloor\omega\rfloor} ; \quad N\left(0,1 ; \mu^{\prime} v^{\prime} ; \omega\right)=\frac{\left\lfloor\omega-\mu^{\prime}\right\rfloor}{\lfloor\omega\rfloor} .
$$

The general expression, equivalent to (2.19), is thereby derived:

$$
N\left(\mu, v ; \mu^{\prime}, v^{\prime} ; \omega\right)=\prod_{r=0}^{\mu-1} \frac{\left\lfloor\omega+v+v^{\prime}-r\right\rfloor}{\lfloor\omega+v-r\rfloor} \prod_{s=0}^{\nu-1} \frac{\left\lfloor\omega-\mu-\mu^{\prime}+s\right\rfloor}{\lfloor\omega-\mu+s\rfloor} .
$$

Finally, using (A.6) and (2.10) one derives the exchange algebras:

$$
\begin{aligned}
V_{1}(\sigma) V^{\mu v}\left(\sigma^{\prime}\right)= & \left(\frac{\Gamma(1+\omega h / \pi) \Gamma((\omega-\mu+v-1) h / \pi)}{\Gamma((\omega-\mu-1) h / \pi) \Gamma(1+(\omega+v) h / \pi)}\right) e^{-i \varepsilon(\mu-v) h / 2} V^{\mu v}\left(\sigma^{\prime}\right) V_{1}(\sigma) \\
& +\left(\frac{\Gamma(1+\omega h / \pi) \Gamma((-\omega+\mu-v+1) h / \pi)}{\Gamma(-v h / \pi) \Gamma(1+(\mu+1) h / \pi)}\right) \\
& \cdot e^{i \varepsilon(2+\mu-v-2 \omega) h / 2} V^{\mu+1 v-1}\left(\sigma^{\prime}\right) V_{2}(\sigma)
\end{aligned}
$$




$$
\begin{aligned}
V_{2}(\sigma) V^{\mu \nu}\left(\sigma^{\prime}\right)= & \left(\frac{\Gamma(1-\omega h / \pi) \Gamma((-\omega+\mu-v-1) h / \pi)}{\Gamma((-\omega-v-1) h / \pi) \Gamma(1+(-\omega+\mu) h / \pi)}\right) e^{-i \varepsilon(\mu-v) h / 2} V^{\mu v}\left(\sigma^{\prime}\right) V_{2}(\sigma) \\
& +\left(\frac{\Gamma(1-\omega h / \pi) \Gamma((\omega-\mu-v+1) h / \pi)}{\Gamma(-\mu h / \pi) \Gamma(1+(v+1) h / \pi)}\right) \\
& \cdot e^{i \varepsilon(2-\mu+v+2 \omega) h / 2} V^{\mu-1 v+1}\left(\sigma^{\prime}\right) V_{1}(\sigma),
\end{aligned}
$$

which through $(\mathrm{A} .14,16,18)$ lead to $(2.17)^{7}$.

Concerning the hermiticity structure discussed in Sect. 3 , it is easily seen that under (3.16), $\left(V^{\mu, v}\right)^{\dagger}=V^{\mu, \nu}$. Applying this relation to (A.14) leads to:

$$
\left(\psi^{\mu, v}\right)^{\dagger}=C^{\mu, v}\left(D^{\mu, v}(\omega-v+\mu)\right)^{\dagger} V^{v, \mu} .
$$

On the the other hand, the $D^{\mu, v}(\omega)$ read, in view of (3.19):

$$
\left.\left.D^{\mu, v}(\omega)=\prod_{t=1}^{\mu-v} \sqrt{\frac{\Gamma(-(\omega-t+1) h / \pi)}{\Gamma((\omega-t) h / \pi)}} \prod_{r=1}^{\mu} \Gamma((\omega-r) h / \pi)\right) \prod_{s=1}^{v} \Gamma((-\omega-s) h / \pi)\right)
$$

if $\mu>v$, and

$$
\left.\left.D^{\mu, v}(\omega)=\prod_{t=1}^{v-\mu} \sqrt{\frac{\Gamma((\omega+t-1) h / \pi)}{\Gamma((-\omega-t) h / \pi)}} \prod_{r=1}^{\mu} \Gamma((\omega-r) h / \pi)\right) \prod_{s=1}^{v} \Gamma((-\omega-s) h / \pi)\right)
$$

if $\mu<v$. They are thus real and a straightforward algebra shows that

$$
D^{\mu, v}(\omega-v+\mu)=D^{v, \mu}(\omega) \text {. }
$$

Since, moreover, the coefficients $C^{v, \mu}$ are symmetric in $\mu$ and $v$, it follows that:

$$
\left(\psi^{\mu, \nu}\right)^{\dagger}=\psi^{v, \mu}
$$

which is equivalent to (3.20).

\section{Appendix B}

In this section we derive Eq. (3.4) by studying the exchange matrix $(1 / 2, J)$ for arbitrary $J$. Considering $\xi_{-1 / 2}^{(1 / 2)} \equiv a_{1} \xi_{1}$, and $\xi_{1 / 2}^{(1 / 2)} \equiv a_{2} \xi_{2}, a_{i}$ normalization constants, we shall determine the general $\xi_{M}^{(J)}$ fields so that the exchange algebra

$$
\xi_{\alpha}^{(1 / 2)}(\sigma) \xi_{M}^{(J)}\left(\sigma^{\prime}\right)=\sum_{M^{\prime}, \alpha^{\prime}}(1 / 2, J)_{\alpha M}^{M^{\prime} \alpha^{\prime}} \xi_{M^{\prime}}^{(J)}\left(\sigma^{\prime}\right) \xi_{\alpha^{\prime}}(\sigma)
$$

has coefficients independent of $\omega$. It is convenient to define

$$
\begin{aligned}
& \rho_{1}^{(J)}(M) \equiv(1 / 2, J)_{-1 / 2 M}^{M-1 / 2} ; \quad \rho_{2}^{(J)}(M) \equiv(1 / 2, J)_{-1 / 2 M}^{M-1} 1 / 2 \\
& \rho_{3}^{(J)}(M) \equiv(1 / 2, J)_{1 / 2 M}^{M 1 / 2} ; \quad \rho_{4}^{(J)}(M) \equiv \frac{a_{1}}{a_{2} \sin (h)}(1 / 2, J)_{1 / 2 M}^{M+1-1 / 2}
\end{aligned}
$$

\footnotetext{
7 Throughout the article we only consider the half circle $0 \leqq \sigma \leqq \pi$ explicitly. In this way, $\varepsilon$ is strictly equal to the sign of its argument, since the latter goes from $-\pi$ to $\pi$. For the other half circle $\varepsilon$ is continued, in agreement with (2.11)
} 
The rest of the calculation will show that $\rho_{2}$ vanishes. The exchange of $\xi_{-1 / 2}^{(1 / 2)}$ with $\xi_{M}^{(J)}$ thus gives

$$
\left.\left.\left.e^{i h / 2}\lfloor J-m+1\rfloor \mid J, \omega+1\right)_{M}^{m-1}=\lfloor\omega+J+m\rfloor \mid J, \omega-1\right)_{M}^{m}-\lfloor\omega\rfloor \rho_{1}^{(J)}(M) \mid J, \omega\right)_{M}^{m} .
$$

The exchange of $\xi_{1 / 2}^{(1 / 2)}$ with $\xi_{M}^{(J)}$ gives

$$
\begin{aligned}
&\left.e^{i h(\omega / 2+m)} \rho_{4}^{(J)}(M)\lfloor\omega\rfloor \mid J, \omega\right)_{M+1}^{m} \\
&=\left.\left.e^{-i h(\omega+1) / 2}\left(e^{i m h}\lfloor\omega+J+m\rfloor \mid J, \omega-1\right)_{M}^{m}-e^{i m h}\lfloor\omega\rfloor \rho_{3}^{(J)}(M) \mid J, \omega\right)_{M}^{m}\right) \\
&\left.-e^{i h(3 \omega / 2+m)}\lfloor J-m+1\rfloor \mid J, \omega+1\right)_{M}^{m-1} .
\end{aligned}
$$

Combining these two relations one obtains a recurrence relation with fixed $m$ :

$$
\begin{aligned}
\left.e^{i h(m+1 / 2)} \rho_{4}^{(J)}(M) \mid J, \omega\right)_{M+1}^{m}= & \left.-2 i e^{i h m}\lfloor\omega+J+m\rfloor \mid J, \omega-1\right)_{M}^{m} \\
& \left.+\frac{1}{\sin (h)}\left(e^{i h(\omega+m)} \rho_{1}^{(J)}(M)-e^{-i h(\omega+m)} \rho_{3}^{(J)}(M)\right) \mid J, \omega\right)_{M}^{m} .
\end{aligned}
$$

Particular cases: The exchange of $\xi_{1 / 2}^{(1 / 2)}$ and $\xi_{-J}^{(J)}$ gives:

$$
\begin{gathered}
\rho_{1}^{(J)}(-J)=e^{-i h J}, \\
\mid J, \omega)_{-J}^{m}=\kappa_{J} e^{-i h m \omega} e^{-i h\left(m^{2}-m / 2\right)}\left(\begin{array}{c}
2 J \\
J+m
\end{array}\right),
\end{gathered}
$$

$\kappa_{J}$ is a normalization constant. Equation (B.5) with $M=-J$ gives

$$
\begin{gathered}
\rho_{3}^{(J)}(-J)=\exp (i h J), \\
\left.\mid J, \omega)_{-J+1}^{m}=\frac{-2 i e^{-i h / 2}}{\rho_{4}(-J)}\left(e^{i h(\omega+m)}\lfloor J+m\rfloor+e^{-i h(\omega+m)}\lfloor J-m\rfloor\right) \mid J, \omega\right)_{-J}^{m},
\end{gathered}
$$

which satisfies Eq. (B.4). Equation (B.6) with $M=-J+1$ gives

$$
\begin{aligned}
& \rho_{1}^{(J)}(-J+1)=e^{i h(-J+1)} ; \quad \rho_{3}^{(J)}(-J+1)=e^{-i h(-J+1)}, \\
\mid J, \omega)_{-J+2}^{m}= & \frac{-2 i e^{-i h / 2}}{\rho_{4}(-J+1)} \frac{-2 i e^{-i h / 2}}{\rho_{4}(-J)}\left(e^{2 i h(\omega+m)}\lfloor J+m\rfloor\lfloor J+m-1\rfloor\right. \\
& \left.\left.+2 \cos (h)\lfloor J+m\rfloor\lfloor J-m\rfloor+e^{-2 i h(\omega+m)}\lfloor J-m\rfloor\lfloor J-m-1\rfloor\right) \mid J, \omega\right)_{-J}^{m} .
\end{aligned}
$$

The above examples lead to the following ansatz for the general solution:

$$
\begin{aligned}
\mid J, \omega)_{-J+P}^{m} & =C(J,-J+P) \mid J, \omega)_{-J}^{m} \sum_{r=-P / 2}^{P / 2} e^{2 i h r(\omega+m)} A_{P}^{r}(J, m), \\
C(J,-J) & =1, \quad A_{0}^{0}=1,
\end{aligned}
$$

$r$ is integer for $P$ even and half integer for $P$ odd. Assume:

$$
C(J, M+1)=\frac{-2 i e^{-i h / 2}}{\rho_{4}^{(J)}(M)} C(J, M),
$$


Eq. (B.6), with $\rho_{1}^{(J)}(M)=e^{i h M}$, and $\rho_{3}^{(J)}(M)=e^{-i h M}$, gives:

$$
\begin{aligned}
A_{P+1}^{r}(J, m)= & e^{i h(P+1 / 2-r)}\left[J+m-r-\frac{P-1}{2}\right] A_{P}^{r-1 / 2}(J, m) \\
& +e^{i h(-P-1 / 2-r)}\left[J-m+r-\frac{P-1}{2}\right] A_{P}^{r+1 / 2}(J, m) .
\end{aligned}
$$

The solution is:

$$
\left.A_{P}^{r}(J, m)=\frac{\lfloor J+m\rfloor !}{\left\lfloor J+m-\frac{P}{2}-r\right\rfloor !\left\lfloor J-m-\frac{P}{2}+r\right\rfloor !\left(\frac{P}{2}+r\right.}\right)
$$

One may verify that $\rho_{4}^{(J)}(M)$ is not determined. Contrary to the other $\rho$ 's, it depends upon the normalization of the $\xi$ 's. The choice appropriate to the quantum group connection is:

$$
\rho_{4}^{(J)}(M)=-2 i e^{i h / 2} \frac{a_{1}}{a_{2}} \sqrt{\lfloor J+M+1\rfloor\lfloor J-M\rfloor}
$$

which leads to

$$
C(J, M)=\left(e^{-i h} \frac{a_{2}}{a_{1}}\right)^{J+M} \sqrt{\frac{\lfloor J-M] !}{[J+M\rfloor !\lfloor 2 J] !}} .
$$

Indeed, with this choice, one has

$$
\begin{aligned}
(1 / 2, J)_{\alpha M}^{M^{\prime} \alpha^{\prime}} & =\left(\left\langle1 / 2, \alpha|\otimes\langle J, M|) \mathbf{R}^{1 / 2, J}\left(\left|J, M^{\prime}\right\rangle \otimes\left|1 / 2, \alpha^{\prime}\right\rangle\right),\right.\right. \\
\mathbf{R}^{1 / 2, J} & =\mathrm{e}^{\left(-2 i h S_{3} J_{3}\right)}\left(1+\left(1-e^{2 i h}\right) e^{-i h S_{3}} S_{+} e^{i h J_{3}} J_{-}\right),
\end{aligned}
$$

where $S_{ \pm}, S_{3}$ correspond to the $J=1 / 2$ representation.

Collecting everything together, one arrives at the remarkably compact formula:

$$
\begin{aligned}
\mid J, \omega)_{M}^{m}= & \left(e^{i h} \frac{a_{2}}{a_{1}}\right)^{J+M} \kappa_{J} \sqrt{\left(\begin{array}{c}
2 J \\
J+M
\end{array}\right)} e^{i h m / 2} \\
& \cdot \sum_{(J-M+m-s) / 2 \text { integer }} e^{i h s(\omega+m)}\left(\begin{array}{c}
J-M \\
(J-M+m-s) / 2
\end{array}\right)\left(\begin{array}{c}
J+M \\
(J+M+m+s) / 2
\end{array}\right) .
\end{aligned}
$$

Applying this formula for $J=1 / 2$ one sees that $\kappa_{1 / 2}=a_{1} e^{i h / 2}$.

\section{Appendix C}

In this appendix we determine the fusion coefficients $\lambda\left(J, M ; J^{\prime}, M^{\prime}\right)$, starting from an explicit computation of $\lambda\left(1 / 2, \pm 1 / 2 ; J^{\prime}, M^{\prime}\right)$. Consider first the fusion of $\xi_{-1 / 2}^{(1 / 2)}$, 
with $\xi_{-J+P}^{(J)}$. Formulae (A.22) combined with (B.4) give

$$
\begin{aligned}
\xi_{-1 / 2}^{(1 / 2)} \xi_{-J+P}^{(J)} \sim & \kappa_{1 / 2} e^{i h / 2} \sum_{m=-J+1}^{J} \psi_{m-1 / 2}^{J+1 / 2} \\
& \cdot\left[\frac{\lfloor\omega+J+m\rfloor}{\lfloor\omega\rfloor}\left\{u_{1}^{1}+u_{1}^{2} e^{-i n / 2} \frac{\lfloor\omega-J+m-1\rfloor}{\lfloor J-m+1\rfloor}\right\} \mid J, \omega-1\right)_{-J+P}^{m} \\
& \left.\left.-\frac{\lfloor\omega-J+m\rfloor}{\lfloor J-m+1\rfloor} e^{i n(P-J-1 / 2)} \mid J, \omega-1\right)_{-J+P}^{m}\right]
\end{aligned}
$$

Substituting the explicit expressions one finds that the coefficient of $\psi_{m-1 / 2}^{J+1 / 2}$ reads:

$$
\begin{gathered}
\kappa_{1 / 2} e^{i h / 2} e^{i h \omega / 2} C(J,-J+P) \frac{\mid J, \omega)_{-J}^{m}}{[J-m+1]} \sum_{r} e^{2 i h r(\omega+m)} e^{i h(m+P / 2-r)} \\
\cdot\left(\lfloor 2 J+1-r-P / 2\rfloor A_{P}^{r}(J, m)+\lfloor P / 2+r+1\rfloor A_{P}^{r+1}(J, m)\right) .
\end{gathered}
$$

From (B.16) one sees that:

$$
\begin{aligned}
& \quad[2 J+1-r-P / 2\rfloor A_{P}^{r}(J, m)+[P / 2+r+1\rfloor A_{P}^{r+1}(J, m) \\
& \quad=[2 J-P+1] A_{P}^{r}(J+1 / 2, m-1 / 2) .
\end{aligned}
$$

Equation (B.8) gives

$$
\left.\left.e^{i h(\omega / 2+m)} \mid J, \omega\right)_{-J}^{m}=\frac{\kappa_{J}}{\kappa_{J+1 / 2}} \frac{e^{i h / 2}}{\lfloor 2 J+1\rfloor} \mid J, \omega\right)_{-J-1 / 2}^{m-1 / 2} .
$$

Using Eq. (B.18) one finally finds:

$$
\lambda(1 / 2,-1 / 2 ; J, M)=\frac{\kappa_{1 / 2} \kappa_{J}}{\kappa_{J+1 / 2}} \sqrt{\frac{\lfloor J-M+1\rfloor}{\lfloor 2 J+1\rfloor}} e^{i h(J+M) / 2} .
$$

A similar calculation based on the identity:

$$
\begin{aligned}
& {[J+1 / 2+m-r-P / 2] A_{P}^{r-1 / 2}(J, m)+\lfloor J+3 / 2-m+r+P / 2\rfloor A_{P}^{r+1 / 2}(J, m)} \\
& \quad=A_{P+1}^{r}(J+1 / 2, m-1 / 2)
\end{aligned}
$$

leads to

$$
\lambda(1 / 2,1 / 2 ; J, M)=\frac{\kappa_{1 / 2} \kappa_{J}}{\kappa_{J+1 / 2}} \sqrt{\frac{\lfloor J+M+1\rfloor}{\lfloor 2 J+1\rfloor}} e^{-i h(J-M) / 2} .
$$

Next the associativity of the OPE expansion gives the relations $(\alpha= \pm 1 / 2)$ :

$$
\lambda(1 / 2, \alpha ; J, M) \lambda\left(1 / 2+J, \alpha+M ; J^{\prime}, M^{\prime}\right)=\lambda\left(J, M ; J^{\prime}, M^{\prime}\right) \lambda\left(1 / 2, \alpha ; J+J^{\prime}, M+M^{\prime}\right) \text {. }
$$

The general solution is

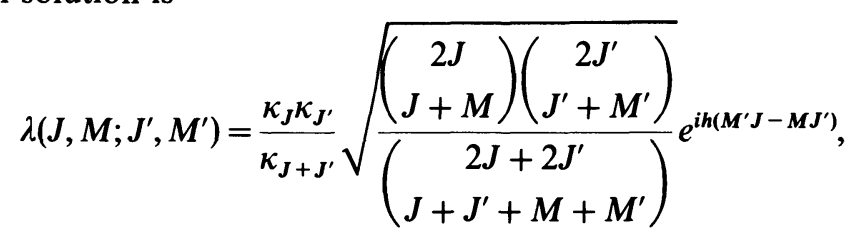


as one easily verifies afterward. It is interesting to note that the second coefficient could be reabsorbed by a change of normalization of the $\xi$ fields, contrary to the third one. This would simplify the fusion coefficients, but spoil the explicit identification of the universal $R$-matrix to be considered next.

\section{Appendix D}

In this section, we determine the general exchange algebra

$$
\xi_{M}^{(J)}(\sigma) \xi_{M^{\prime}}^{\left(J^{\prime}\right)}\left(\sigma^{\prime}\right)=\sum_{N^{\prime} N}\left(J, J^{\prime}\right)_{M M^{\prime}}^{N^{\prime} N} \xi_{N^{\prime}}^{\left(J^{\prime}\right)}\left(\sigma^{\prime}\right) \xi_{N}^{(J)}(\sigma)
$$

from the requirement that the order between fusion and exchange should be irrelevant: consider $(\alpha, \beta= \pm 1 / 2)$

$$
\xi_{\alpha}^{(1 / 2)}(x) \xi_{M-\alpha}^{(J)}(\sigma) \xi_{M^{\prime}}^{\left(J^{\prime}\right)}\left(\sigma^{\prime}\right)=\sum_{R N N^{\prime} \beta}\left(J, J^{\prime}\right)_{M-\alpha M^{\prime}}^{R N-\beta}\left(1 / 2, J^{\prime}\right)_{\alpha R}^{\beta N^{\prime}} \xi_{N^{\prime}}^{\left(J^{\prime}\right)}\left(\sigma^{\prime}\right) \xi_{\beta}^{(1 / 2)}(x) \xi_{N-\beta}^{(J)}(\sigma)
$$

Letting $x \rightarrow \sigma$, and using (C.9) one gets

$$
\lambda(1 / 2, \alpha ; J, M-\alpha)\left(J+1 / 2, J^{\prime}\right)_{M M^{\prime}}^{N^{\prime} N}=\sum_{R N N^{\prime} \beta}\left(J, J^{\prime}\right)_{M-\alpha M^{\prime}}^{R N-\beta}\left(1 / 2, J^{\prime}\right)_{\alpha R}^{\beta N^{\prime}} \lambda(1 / 2, \beta ; J, N-\beta) .
$$

This gives:

$$
\begin{aligned}
& \left(J+1 / 2, J^{\prime}\right)_{M M^{\prime}}^{N^{\prime} N}=\frac{\lambda(1 / 2,-1 / 2 ; J, N+1 / 2)}{\lambda(1 / 2,-1 / 2 ; J, M+1 / 2)} e^{i h N^{\prime}}\left(J, J^{\prime}\right)_{M+1 / 2}^{N^{\prime} N+1 / 2}, \\
& \left(J+1 / 2, J^{\prime}\right)_{M M^{\prime}}^{N^{\prime} N}=\frac{\lambda(1 / 2,1 / 2 ; J, N-1 / 2)}{\lambda(1 / 2,1 / 2 ; J, M-1 / 2)} e^{-i h N^{\prime}}\left(J, J^{\prime}\right)_{M-1 / 2}^{N^{\prime} N-1 / 2} \\
& +\frac{\lambda(1 / 2,-1 / 2 ; J, N+1 / 2)}{\lambda(1 / 2,1 / 2 ; J, M-1 / 2)} \rho_{4}^{\left(J^{\prime}\right)}\left(N^{\prime}-1\right)\left(J, J^{\prime}\right)_{M-1 / 2}^{N^{\prime}-1} M^{\prime},
\end{aligned}
$$

which reduces to:

$$
\begin{aligned}
\left(J+1 / 2, J^{\prime}\right)_{M M^{\prime}}^{N^{\prime} N}= & e^{i h\left(N^{\prime}+N / 2-M / 2\right)} \sqrt{\frac{\lfloor J-N+1 / 2\rfloor}{\lfloor J-M+1 / 2\rfloor}}\left(J, J^{\prime}\right)_{M+1 / 2}^{N^{\prime} N+1 / 2}, \\
\left(J+1 / 2, J^{\prime}\right)_{M M^{\prime}}^{N^{\prime} N}= & e^{i h\left(N^{\prime}+N / 2-M / 2\right)} \sqrt{\frac{\lfloor J+N+1 / 2\rfloor}{\lfloor J+M+1 / 2\rfloor}}\left(J, J^{\prime}\right)_{M-1 / 2 M^{\prime}}^{N^{\prime} N-1 / 2} \\
& +\left(1-e^{2 i h}\right) e^{i h(J+1 / 2+N-M)} \\
& \cdot \sqrt{\frac{\lfloor J+N+1 / 2\rfloor}{\lfloor J+M+1 / 2\rfloor}\left\lfloor J+N^{\prime}\right\rfloor\left\lfloor J-N^{\prime}+1\right\rfloor\left(J, J^{\prime}\right)_{M-1 / 2 M^{\prime}}^{N^{\prime}-1 / 2}} .
\end{aligned}
$$

These equations are solved by introducing the ansatz:

$$
\left(J, J^{\prime}\right)_{M M^{\prime}}^{N^{\prime} N}=\left(\left\langleJ, M\left|\otimes\left\langle J^{\prime}, M^{\prime}\right|\right) \mathbf{R}\left(|J, N\rangle \otimes\left|J^{\prime}, N^{\prime}\right\rangle\right),\right.\right.
$$




$$
\mathbf{R}=e^{\left(-2 i h J_{3} \otimes J_{3}\right)}\left(1+\sum_{n=1}^{\infty} \beta_{n} e^{-i h n J_{3}}\left(J_{+}\right)^{n} \otimes e^{i h n J_{3}}\left(J_{-}\right)^{n}\right),
$$

where the $\beta_{n}$ 's are constants to be determined. Using (3.14), one sees that this formula precisely solves (D.6) for arbitrary $\beta_{n}$. Equation (D.7) leads to:

$$
\beta_{n}=\beta_{n-1} e^{i h(n-1)}\left(1-e^{2 i h}\right) /\lfloor n\rfloor .
$$

Equation (B.20) shows that $\beta_{1}=1-e^{2 i h}$. Thus

$$
\beta_{n}=\frac{\left(1-e^{2 i n}\right)^{n} e^{i h n(n-1) / 2}}{\lfloor n\rfloor !} \text { Q.E.D. }
$$

\section{Appendix E}

In this appendix, we give some details about Sect. 4. First let us generalize the discussion of Appendix A. Remember Eq. (A.11a, b). Using again (A.6) and (2.11) one derives similar relations:

$$
\begin{aligned}
& V_{1}(\sigma) \hat{V}^{\hat{\mu}, \hat{v}}\left(\sigma^{\prime}\right) \sim\left(\frac{\Gamma(1+\omega h / \pi) \Gamma(1+h / \pi+\hat{\mu}+\hat{v})}{\Gamma(1+\omega h / \pi+\hat{v}) \Gamma(1+h / \pi+\hat{\mu})}\right) V^{1,0 ; \hat{\mu}, \hat{v}}(\sigma), \\
& V_{2}(\sigma) \hat{V}^{\hat{\mu}, \hat{v}}\left(\sigma^{\prime}\right)=\left(\frac{\Gamma(1-\omega h / \pi) \Gamma(1+h / \pi+\hat{\mu}+\hat{v})}{\Gamma(1-\omega h / \pi+\hat{\mu}) \Gamma(1+h / \pi+\hat{v})}\right) V^{0,1 ; \hat{\mu}, \hat{v}}(\sigma) .
\end{aligned}
$$

We have introduced $\hat{V}^{\hat{\alpha} \beta}$ following the general convention. Working out the recurrence formulae similar to (A.15-18), one finds that

$$
\psi_{m \hat{m}}^{(J \hat{J})}(\sigma) \equiv C^{\mu, v ; \hat{\mu}, \hat{v}} D^{\mu, v ; \hat{\mu}, \hat{v}}(\omega) V^{\mu, v ; \hat{\mu}, \hat{v}}(\sigma),
$$

where $2 J=\mu+v, 2 m=v-\mu, 2 \hat{J}=\hat{\mu}+\hat{v}, 2 \hat{m}=\hat{v}-\hat{\mu}$, and

$$
C^{\mu, v ; \hat{\mu}, \hat{v}}=C^{\mu, v} \hat{C}^{\hat{\mu}, \hat{v}} \frac{\prod_{r=1}^{\mu+v} \prod_{\hat{r}=1}^{\hat{\mu}+\hat{v}}(r \sqrt{h / \pi}+\hat{r} \sqrt{\pi / h})}{\prod_{s=1}^{\mu} \prod_{\hat{s}=1}^{\hat{\mu}}(s \sqrt{h / \pi}+\hat{s} \sqrt{\pi / h}) \prod_{t=1}^{v} \prod_{\hat{t}=1}^{\hat{v}}(s \sqrt{h / \pi}+\hat{s} \sqrt{\pi / h})},
$$

and choosing, for instance, $\mu>v, \hat{\mu}>\hat{v}$,

$$
D^{\mu, v ; \hat{\mu}, \hat{v}}=D^{\mu, v} \hat{D}^{\hat{\mu}, \hat{v}} \frac{\prod_{t=1}^{\mu-v \hat{\mu}-\hat{v}} \prod_{\hat{i}=1}^{\mu}(-(\omega+t-1) \sqrt{h / \pi}-(\hat{t}-1) \sqrt{\pi / h})((\omega-t) \sqrt{h / \pi}-\hat{t} \sqrt{\pi / h})}{\prod_{r=1}^{\mu} \prod_{\hat{r}=1}^{\hat{\mu}}((\omega-r) \sqrt{h / \pi}-\hat{r} \sqrt{\pi / h}) \prod_{s=1}^{\mu} \prod_{\hat{s}=1}^{\hat{v}}(-(\omega+s) \sqrt{h / \pi}-\hat{s} \sqrt{\pi / h})} .
$$

All these formulae are invariant under $h \rightarrow \hat{h}$ if one lets $\omega \rightarrow \hat{\omega}=\omega h / \pi, \mu \leftrightarrow \hat{\mu}$, and $v \leftrightarrow \hat{v}$.

The recursion for the generalized fusion coefficients is determined by

$$
N(1,0,0,0 ; \mu, v, \hat{\mu}, \hat{v} ; \omega)=(-1)^{\hat{v}} N(1,0 ; \mu, v ; \omega),
$$




$$
\begin{aligned}
& N(0,0,1,0 ; \mu, v, \hat{\mu}, \hat{v} ; \omega)=(-1)^{v} \hat{N}(1,0 ; \hat{\mu}, \hat{v} ; \hat{\omega}) \\
& N(0,1,0,0 ; \mu, v, \hat{\mu}, \hat{v} ; \omega)=(-1)^{\hat{\mu}} N(0,1 ; \mu, v ; \omega) \\
& N(0,0,0,1 ; \mu, v, \hat{\mu}, \hat{v} ; \omega)=(-1)^{\mu} \hat{N}(0,1 ; \hat{\mu}, \hat{v} ; \hat{\omega})
\end{aligned}
$$

obtaining

$$
\begin{aligned}
N\left(\mu, v, \hat{\mu}, \hat{v} ; \mu^{\prime}, v^{\prime}, \hat{\mu}^{\prime}, \hat{v}^{\prime} ; \omega\right)= & (-1)^{\hat{v}^{\prime} \mu+\hat{\mu}^{\prime} v+v^{\prime} \hat{\mu}+\mu^{\prime} \hat{v}} \\
& \cdot N\left(\mu, v ; \mu^{\prime}, v^{\prime} ; \omega\right) \hat{N}\left(\hat{\mu}, \hat{v} ; \hat{\mu}^{\prime}, \hat{v}^{\prime}, \omega\right),
\end{aligned}
$$

which is equivalent to (4.5).

Consider next the exchange. Making use of (A.6) and (2.10) one derives

$$
\begin{gathered}
V_{1}(\sigma) \hat{V}^{\hat{\mu} \hat{v}}\left(\sigma^{\prime}\right)=\left(\frac{\Gamma(1+\omega h / \pi) \Gamma((\omega-1) h / \pi-\hat{\mu}+\hat{v})}{\Gamma((\omega-1) h / \pi-\hat{\mu}) \Gamma(1+\omega h / \pi+\hat{v})}\right) e^{i \varepsilon(\hat{\mu}-\hat{v}) \pi / 2} \hat{V}^{\hat{\mu}, \hat{v}}\left(\sigma^{\prime}\right) V_{1}(\sigma) \\
V_{2}(\sigma) \hat{V}^{\hat{\mu} \hat{v}}\left(\sigma^{\prime}\right)=\left(\frac{\Gamma(1-\omega h / \pi) \Gamma(-(\omega+1) h / \pi+\hat{\mu}-\hat{v})}{\Gamma(-(\omega+1) h / \pi-\hat{v}) \Gamma(1-\omega h / \pi+\hat{\mu})}\right) e^{i \varepsilon(\hat{\mu}-\hat{v}) h / 2} \hat{V} \hat{\mu}, \hat{v}\left(\sigma^{\prime}\right) V_{2}(\sigma) .
\end{gathered}
$$

Due to Eq. (4.1), that is $h \hat{h}=\pi^{2}$, in each formula there is only one term since the second one involves the inverse of a $\Gamma$-function with a negative integer argument. Consider the first relation as an example. One derives an exchange algebra for the $\psi$ fields after multiplying both sides by $d_{1}(\omega) \hat{C}^{\hat{\mu} \hat{v}} \hat{D}^{\hat{\mu} \hat{v}}(\hat{\omega}-\pi / h)$. From the above discussion, we know that

$$
\left.\left.\hat{D}^{\hat{\mu} \hat{v}}(\hat{\omega})=\prod_{\hat{i}=0}^{\hat{\mu}-\hat{v}} \sqrt{\frac{\Gamma((-\hat{\omega}+\hat{t}-1) \hat{h} / \pi)}{\Gamma((\hat{\omega}-\hat{t}) \hat{h} / \pi)}} \prod_{\hat{r}=0}^{\hat{\mu}} \Gamma((\hat{\omega}-\hat{r}) \hat{h} / \pi)\right) \prod_{\hat{s}=0}^{\hat{v}} \Gamma(-(\hat{\omega}+\hat{s}) \hat{h} / \pi)\right),
$$

where we took $\hat{\mu}>\hat{v}$ as an example. This may be transformed into:

$$
\hat{D}^{\hat{\mu} \hat{v}}(\hat{\omega})=\prod_{\hat{t}=0}^{\hat{\mu}-\hat{v}} \sqrt{\frac{\Gamma(-\omega+(\hat{t}-1) \pi / h)}{\Gamma(\omega-\hat{t} \pi / h)}} \prod_{\hat{r}=0}^{\hat{\mu}} \Gamma(\omega-\hat{r} \pi / h) \prod_{\hat{s}=0}^{\hat{v}} \Gamma(-\omega+\hat{s} \pi / h) .
$$

It follows from (4.2) $(\hat{\omega}=\omega \pi / h)$ that $\omega$ is shifted by 1 when $\hat{\omega}$ is shifted by $\pi / h$. It is thus clear on the last equation that $\hat{D}^{\hat{\mu} \hat{v}}(\hat{\omega})$ and $\hat{D}^{\hat{\mu} \hat{v}}(\hat{\omega}-\pi / h)$ may be related using the relation $\Gamma(z+1)=z \Gamma(z)$. After some algebra one arrives at the very simple relation

$$
\psi_{1}(\sigma) \hat{\psi}_{\hat{m}}^{(\hat{J})}\left(\sigma^{\prime}\right)=e^{-i \pi \hat{J}_{\varepsilon}} \hat{\psi}_{\hat{m}}^{(\hat{J})}\left(\sigma^{\prime}\right) \psi_{1}(\sigma),
$$

which is also true if $\hat{\mu}<\hat{v}$. A similar calculation, starting from (E.1b) leads to:

$$
\psi_{2}(\sigma) \hat{\psi}_{\dot{m}}^{(\hat{J})}\left(\sigma^{\prime}\right)=e^{-i \pi \hat{J} \varepsilon} \hat{\psi}_{\dot{m}}^{(\hat{J})}\left(\sigma^{\prime}\right) \psi_{2}(\sigma) .
$$

Next one extends these formulae to the general $\psi_{\hat{m}}^{\hat{J}}(\sigma)$ operator by fusion. Using the fact that the fusion coefficients (A.23) are products of terms of the form $\lfloor\omega+a\rfloor /\lfloor\omega+b\rfloor, a$ and $b$, constants, which satisfy, for any integer $r$,

$$
\frac{\lfloor\omega+r \pi / h+a\rfloor}{\lfloor\omega+r \pi / h+b\rfloor}=\frac{\lfloor\omega+a\rfloor}{\lfloor\omega+b\rfloor}
$$


one finds

$$
\psi_{m}^{(J)}(\sigma) \hat{\psi}_{\hat{m}}^{(\hat{J})}\left(\sigma^{\prime}\right)=e^{-2 i \pi J \hat{J}_{\varepsilon}} \hat{\psi}_{\dot{m}}^{(\hat{J})}\left(\sigma^{\prime}\right) \psi_{m}^{(J)}(\sigma) .
$$

Thus the exchange algebra between $\psi$ 's and $\hat{\psi}$ 's is trivial.

This is extended to the $\xi$-fields if one multiplies the last equation by

$$
\left.\mid J, \omega)_{M}^{m} \mid \widehat{J, \hat{\omega}-m \pi / h}\right)_{\hat{M}}^{\hat{m}},
$$

and sums over $m$ and $\hat{m}$. The crucial point for this is that the explicit expression (B.21) is such that, for any integer $r$,

$$
\left.\mid J, \omega+r \pi / h)_{M}^{m}=e^{i \pi r(J-M+m)} \mid J, \omega\right)_{M}^{m} .
$$

Acknowledgements. This work was partially done while I was attending the workshop on the mathematics of conformal field theories at the Aspen Center of Physics, during the summer 1989. I am grateful for the warm hospitality extended to me there. Stimulating discussions with other participants have been helpful.

\section{References}

1. Drinfeld, V.: Hopf algebra and quantum Yang-Baxter equation. Dokl. Akad. Nauk. SSSR 283, 1060-1064 (1985); Jimbo, M.: A $q$-analog of $U\left(g l_{N+1}\right)$ Hecke algebra and the Yang-Baxter equation. Lett. Math. Phys. 11, 247-252 (1985); Faddeev, L., Reshetikhin, N. Yu, Takhtadjan, M. I.: Quantization of Lie groups and Lie algebras, LOMI preprint E-14-87 Leningrad

2. Gervais, J.-L., Neveu, A.: Novel triangle relation and absence of tachyon in Liouville string field theory. Nucl. Phys. B238, 125 (1984)

3. Babelon, O.: Extended conformal algebra and Yang-Baxter equation. Phys. Lett. B215, 523-529 (1988)

4. Gervais, J.-L., Neveu, A.: New quantum treatment of the Liouville field theory. Nucl. Phys. B224, 329-348 (1983)

5. For more recent reviews see: Gervais, J.-L.: Systematic approach to conformal theories. Nucl. Phys. B (Proc. Supp.) 5B, 119-136 (1988) 119; Bilal, A., Gervais, J.-L.: Conformal theories with non-linearly-extended Virasoro symmetries and Lie-algebra classification, Conference Proceedings: "Infinite dimensional Lie algebras and Lie groups." Kac, V. ed. Marseille 1988. Singapore: World-Scientific

6. For generalization to arbitrary groups see: Bilal, A. Gervais, J.-L.: Systematic approach to conformal systems with extended Virasoro symmetries. Phys. Lett. B206, 412-420 (1988); Extended $C=\infty$ conformal systems from classical Toda field theories. Nucl. Phys. B314, 646-686 (1989); Systematic construction of conformal theories with higher-spin Virasoro symmetries. Nucl. Phys. B318, 579-630 (1989)

7. Khono, T.: Monodromy representations of Braid groups and Yang-Baxter equations. Ann. Inst. Fourier 37, 4-21 (1987); Quantized universal enveloping algebras and monodromy of braid groups. Nagoya (Math) preprint 1989 No. 3; Moore, G., Seiberg, N.: Polynomial equations for rational conformal field theories. Phys. Lett. 212, 451-460 (1988); Rehren, K., Schroer, B.: Einstein causality and Artin's braids, Berlin preprint FH-88-0439

8. Felder, G., Fröhlich, J., Keller, G.: Braid matrices and structure constants for minimal conformal models. Commun. Math. Phys. 124, 646-664 (1989)

9. Gervais, J.-L., Neveu, A.: Dual string spectrum in Polyakov's quantization. Nucl. Phys. B209, 125-145 (1982)

10. Belavin, A., Polyakov, A. M., Zamolodchikov, A. B.: Infinite conformal symmetry in twodimensional quantum theory. Nucl. Phys. B241, 333-380 (1984) 
11. Gervais, J.-L., Neveu, A.: Non-standard 2D critical systems from Liouville theory. Nucl. Phys. B257 [FS14] 59-76 (1985)

12. Gervais, J.-L., Neveu, A.: Oscillator representation of 2D-conformal algebra. Commun. Math. Phys. 100, 15-21 (1985)

13. Gervais, J.-L., Neveu, A.: Locality in strong coupling Liouville field theory and string models for dimensions 7, 13 and 19. Phys. Lett. 151B, 271-274 (1985)

14. Leclair, A.: Integrable restrictions quantum soliton theory and minimal models. Cornell preprint PUPT-1124, 1989

15. Gervais, J.-L., Rostand, B.: Two dimensional gravity and its $W_{3}$ extension: strongly coupled unitary theories, Paris preprint LPTENS 89/23

Communicated by K. Gawedzki

Received October 6, 1989; in revised form December 14, 1989

Note added in proof. Since this article was written, the research program which is discussed in the conclusion has been fulfilled to a large extent. This will be the subject of forthcoming publications. 
